Supplement of Biogeosciences, 15, 4495-4513, 2018 https://doi.org/10.5194/bg-15-4495-2018-supplement (C) Author(s) 2018. This work is distributed under the Creative Commons Attribution 4.0 License.

(c) (1)

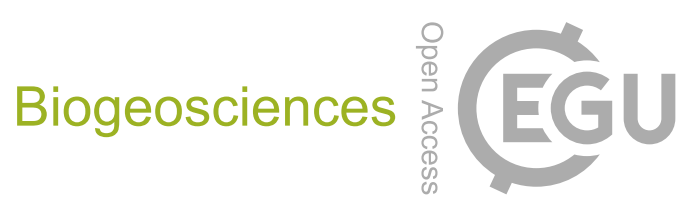

Supplement of

\title{
Does predictability of fluxes vary between FLUXNET sites?
}

Ned Haughton et al.

Correspondence to: Ned Haughton (ned@nedhaughton.com)

The copyright of individual parts of the supplement might differ from the CC BY 4.0 License. 


\section{Supplement for: How unique are fluxes from different FLUXNET sites?}

\section{${ }_{4}$ Aridity}

5 Arid sites tend to have higher precipitation variability, with fewer, heavier rain events, and longer dry periods

(Donat et al., 2016). We would expect that flux predictability would be lower at arid sites. For this hypothesis, we used an aridity index based on mean annual precipitation from CRU TS4.01, and the energy-only estimate for potential evapotranspiration (PET) from Milly and Dunne (2016), based on net radiation and ground heat flux $(\mathrm{PET}=0.8(\overline{\text { Rnet }}-\overline{Q g}))$ from FLUXNET, such that the aridity index $(\mathrm{AI})=$ mean precipitation/PET. 10 We assumed $\mathrm{Qg}=0$ where sites did not provide $\mathrm{Qg}$ (which is approximately true on long time scales).

11 Figure 1 shows the predictability metrics for aridity index. The pattern shown for each flux, and particularly

12 NEE and Qle, is quite similar to that for mean precipitation in Figure 3 in the paper, with more very arid 13 sites being less predictable.

\section{${ }_{14} \quad$ Extended Budyko Analysis}

15 The following 2 plots show predictability metrics for Potential and Actual evapotranspiration. 


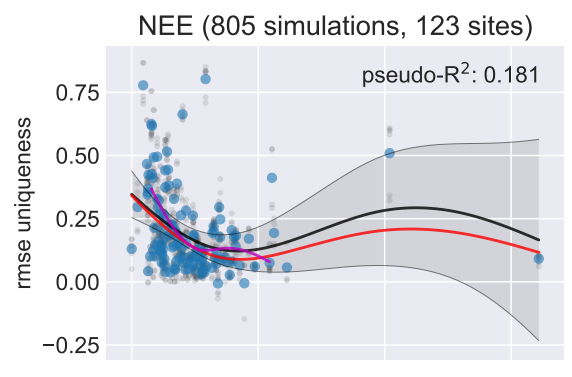

Qh (898 simulations, 123 sites)
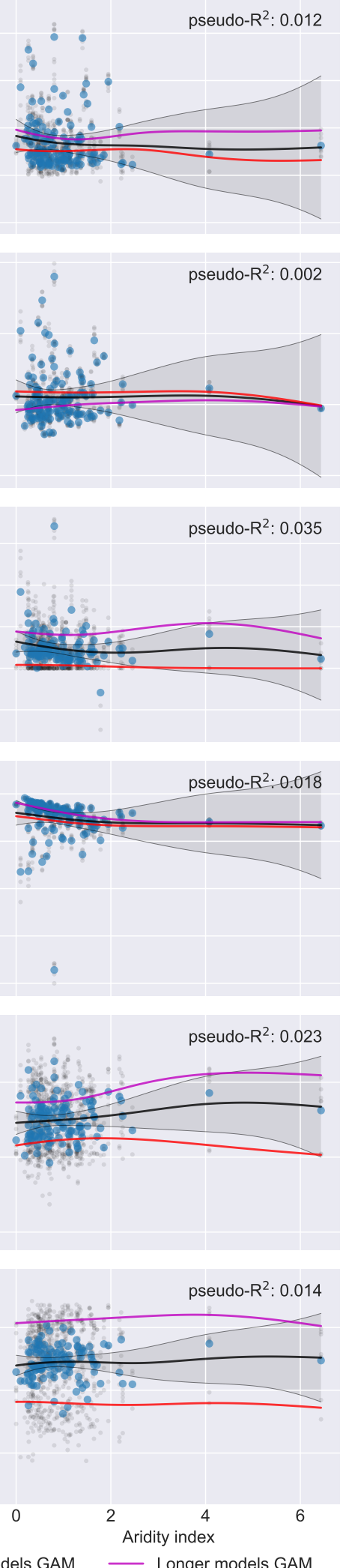

Qle (888 simulations, 123 sites)
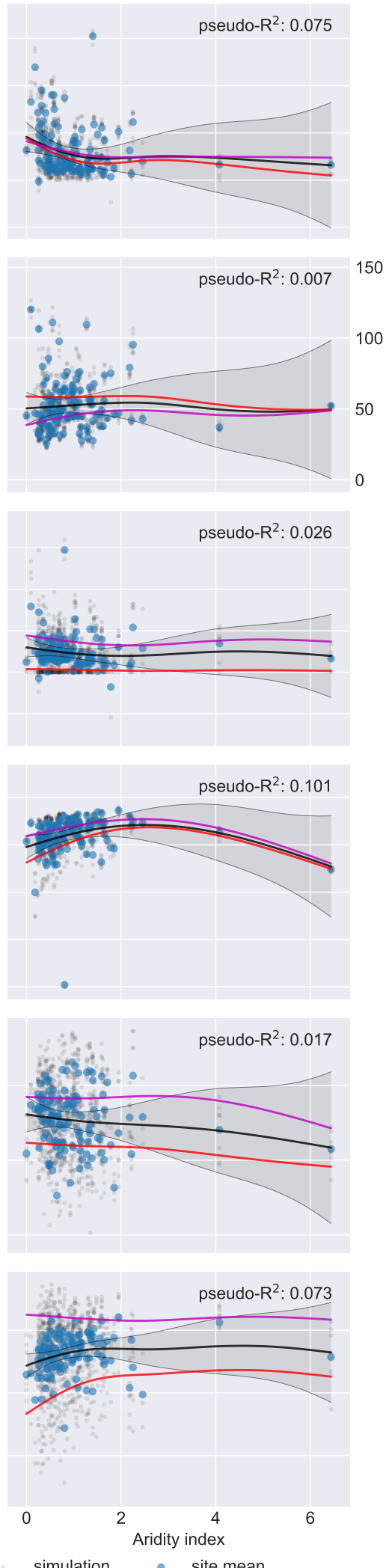

Figure 1: Predictability metrics for Aridity Index. 32 sites failed the Aridity Index calculation. 

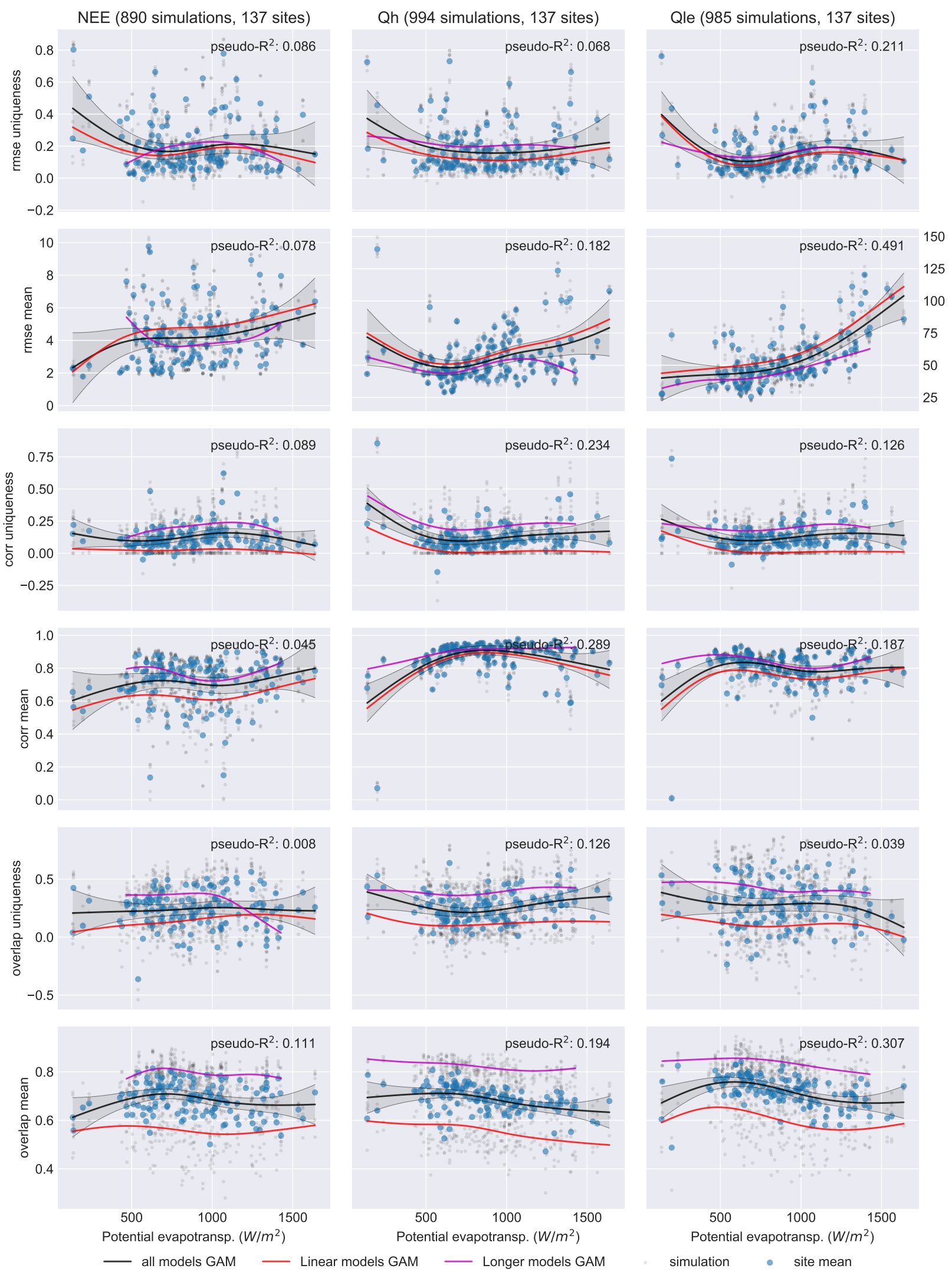

Predictability metrics for Potential Evapotranspiration (PET). 

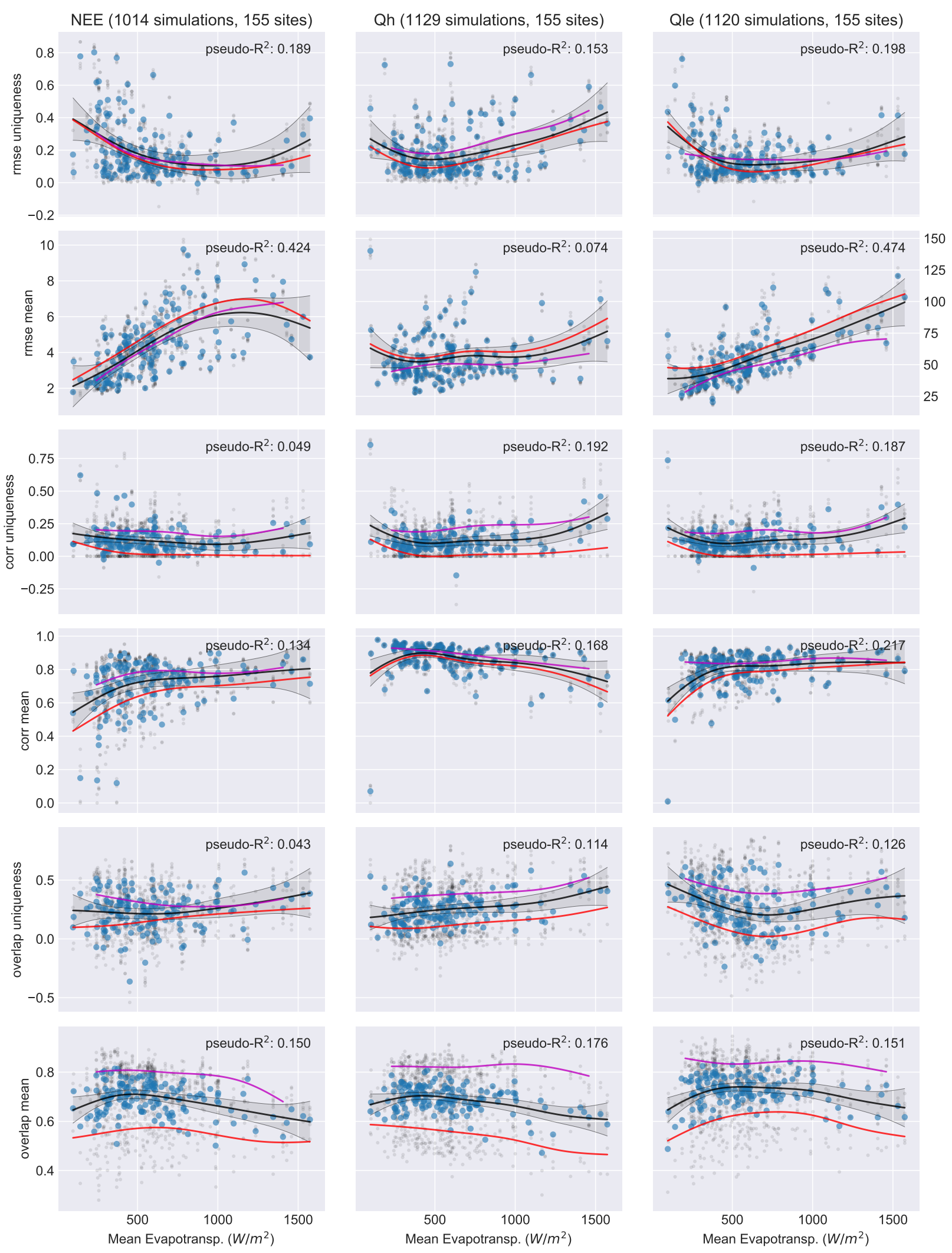

- all models GAM — Linear models GAM — Longer models GAM

Predictability metrics for Actual Evapotranspiration. 


\section{Predictability as a function of site variability}

Interannual variability Sites heavily influenced by longer term climate patterns, such as decadal scale ocean oscillations, are less likely to have all of their relevant patterns captured within the period of FLUXNET measurement, and so potentially contain systematic biases. We compared the interannual variability between sites for both T and P, using the CRU TS4.01 data. We calculated the coefficient of variance (CoV) for annual means of temperature $(\mathrm{K})$, and precipitation (mm/year). We would expect that as IAV increases (shown by greater $\mathrm{CoV})$, predictability would decline.

Seasonality Larger differences between winter and summer conditions would likely lead to lower predictability, since we would expect flux behaviour at such sites to be more diverse over the course of the year. This would also affect the relative influence of time varying factors, e.g. timing of snow melt, or vegetation phenology. For model and site combinations where the training and testing data is more disjointed, this might also lead to lower predictability due to the non-training testing data diverging more in behaviour. Since about $55 \%$ of sites in Tier 1 are less than 5 years long, we used the BioClim variables (WorldClim, 2016) to compare seasonality between sites. We investigated: isothermality - the ratio of diurnal temperature range to annual temperature range; temperature seasonality - the standard deviation of monthly average temperatures, normalised by the annual average in $\mathrm{K}$; temperature annual range; precipitation seasonality; precipitation of wettest quarter; and precipitation of the driest quarter.

Diurnal ranges Sites with large diurnal ranges have stronger rates of change between daily peaks and troughs, and these are likely to make prediction harder. Faster changes in temperature, for example, can cause rapid changes in relative humidity, which is a major driver of latent heat flux. We used the BioClim (WorldClim, 2016) mean diurnal temperature range using the nearest neighbouring grid cell for each site.

\section{Results}

Variability of forcing variables is a major component in the predictability of fluxes. In general, we might expect higher variability to lead to lower predictability. Here we examine predictability at various time scales. Figure 2 shows the predictability metrics over the interannual variability of temperature, and Figure 3 shows the same for precipitation, using the CRU TS4.01 data. There does not appear to be a strong trend in increased predictability with higher interannual temperature variability Figure 2. However, there does appear to be a clear trend toward higher uniqueness at sites with stronger interannual variability in precipitation for NEE and for Qle (Figure 3).

Other modes of variability descending in scale include intra-annual variability, such as annual range, or variance of monthly values (seasonality); means of particular seasons; and diurnal ranges, as well as mixedscale measurements, such as isothermality (ratio of diurnal range to annual range of temperature). Measures of each of these for both temperature and precipitation are included in the BioClim data, and plots of uniqueness as a function of each variable are included in the next section. For the majority of cases, there appears to be no clear patterns of note. The exception includes some increase in RMSE uniqueness in NEE, and perhaps also for Qle, for sites with a higher diurnal temperature range (Figure 5).

\section{Extended BioClim plots}

The following figures use the WorldClim BioClim variables.

- Figure 5 shows the remaining predictability metrics for diurnal temperature range,

- Figure 6 shows isothermality,

- Figure 7 shows temperature seasonality,

- Figure 8 temperature annual range,

- Figure 9 precipitation seasonality,

- Figure 10 precipitation of wettest quarter, and

- Figure 11 precipitation of the driest quarter. 


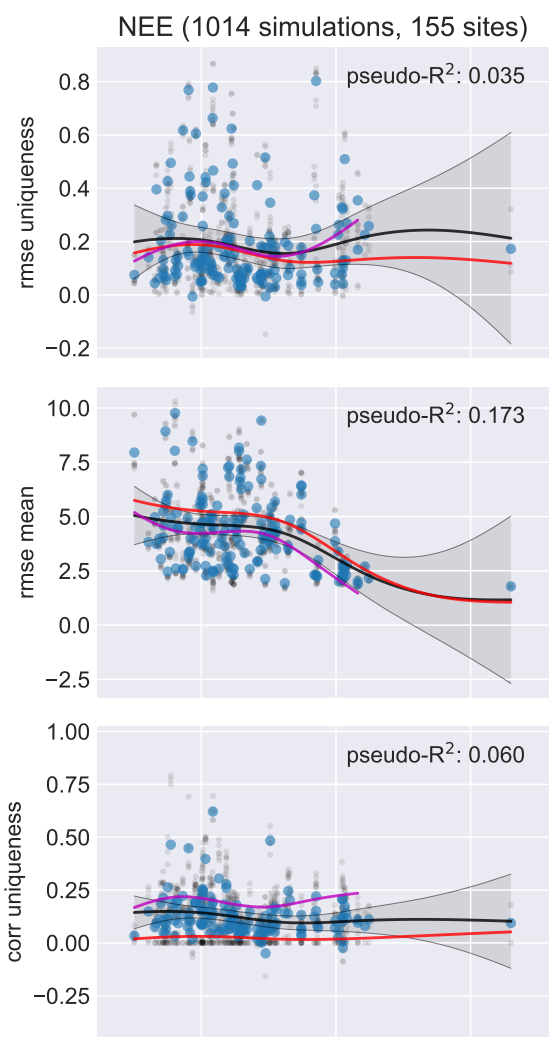

Qh (1129 simulations, 155 sites)
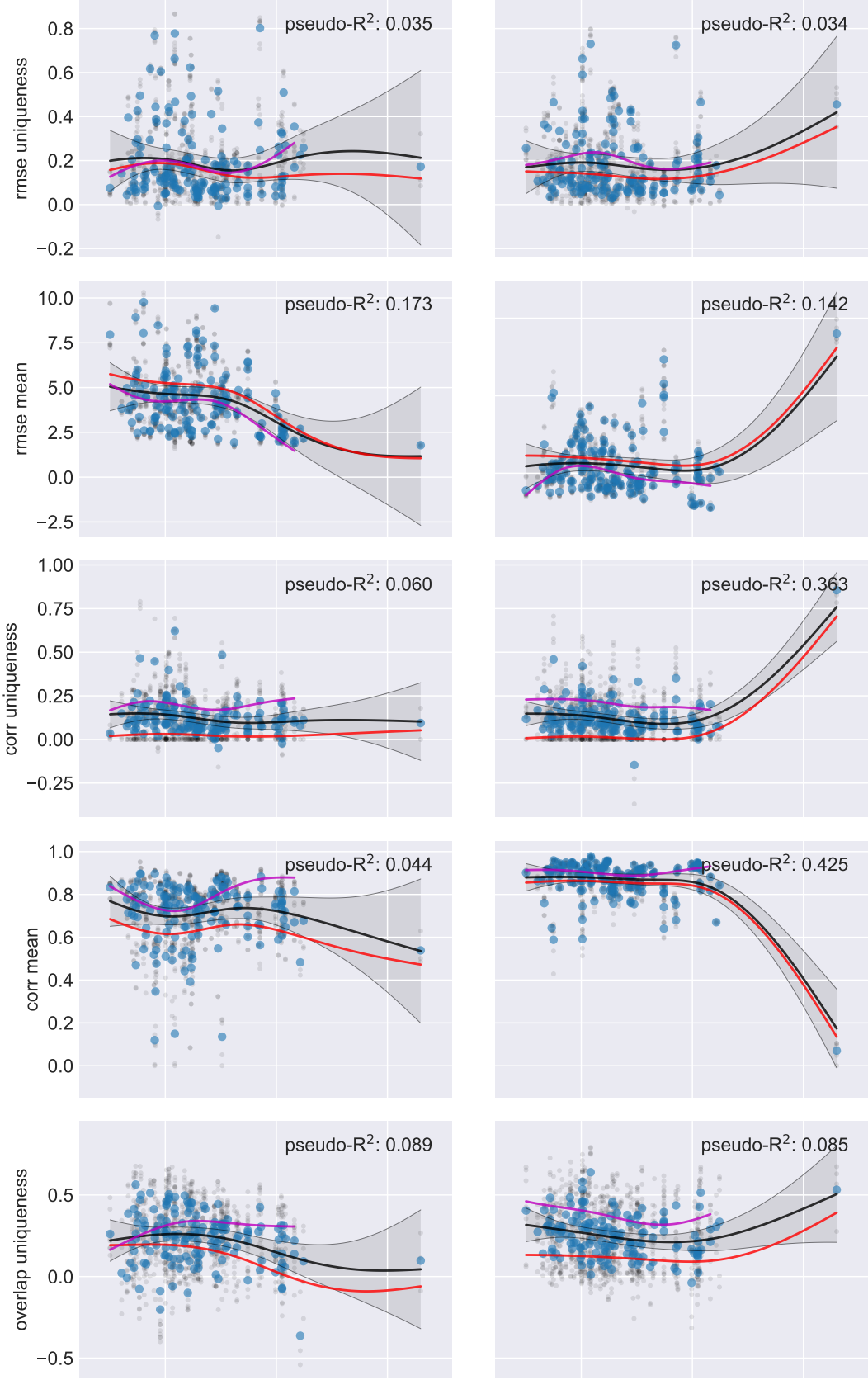
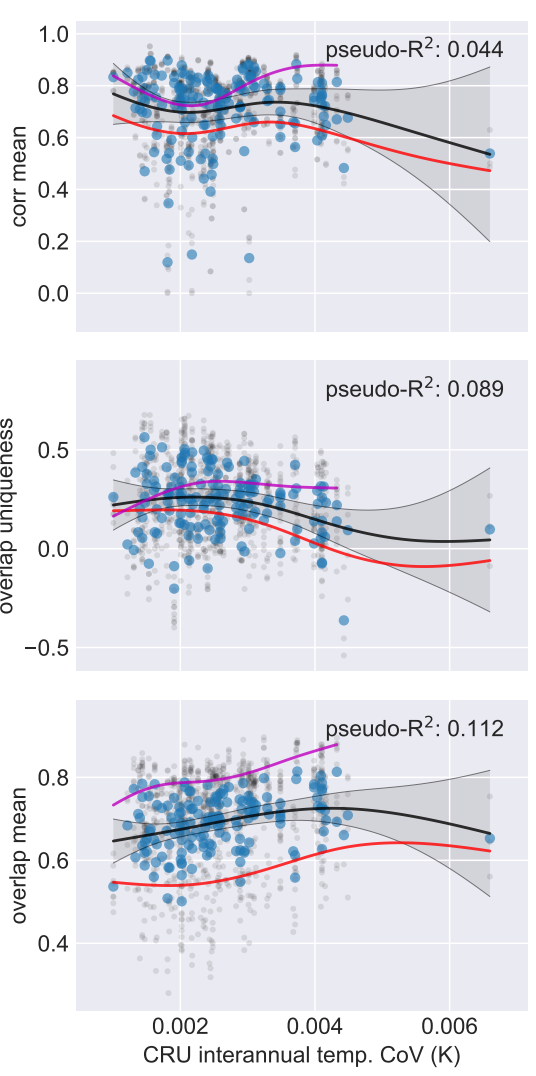

$\mathrm{CoV}(\mathrm{K})$

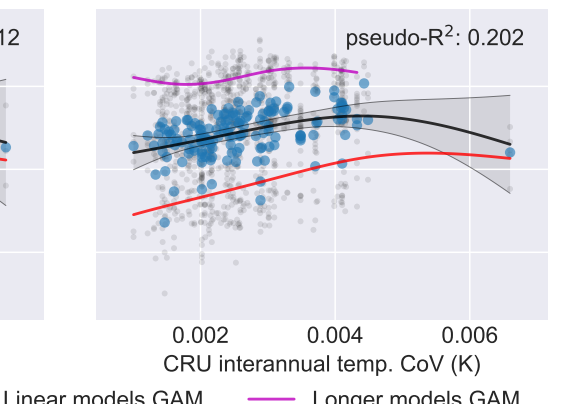

Qle (1120 simulations, 155 sites)
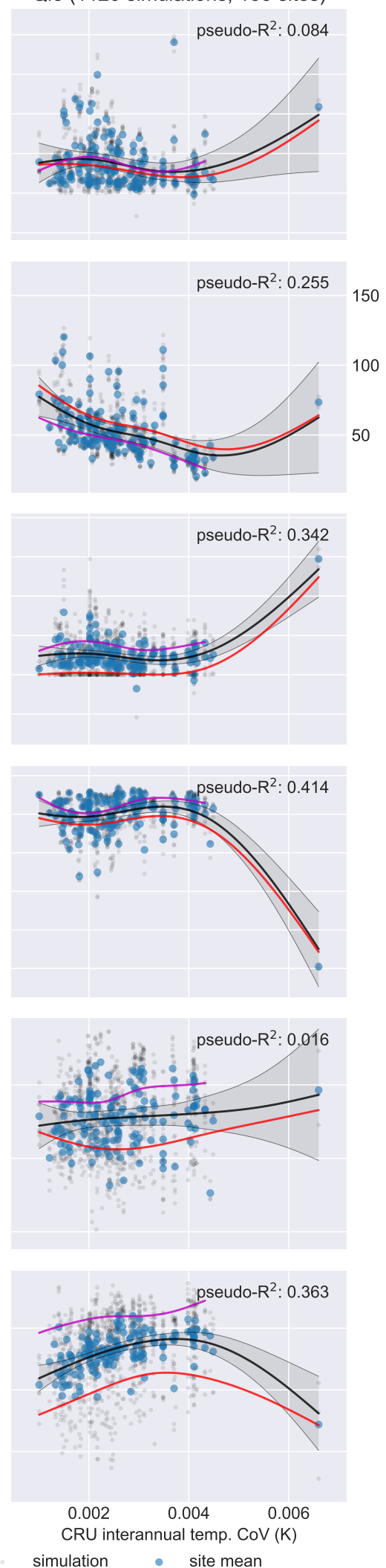

Figure 2: RMSE predictability by temperature interannual variability, calculated from the coefficient of variation in the CRU TS4.01 annual means. 

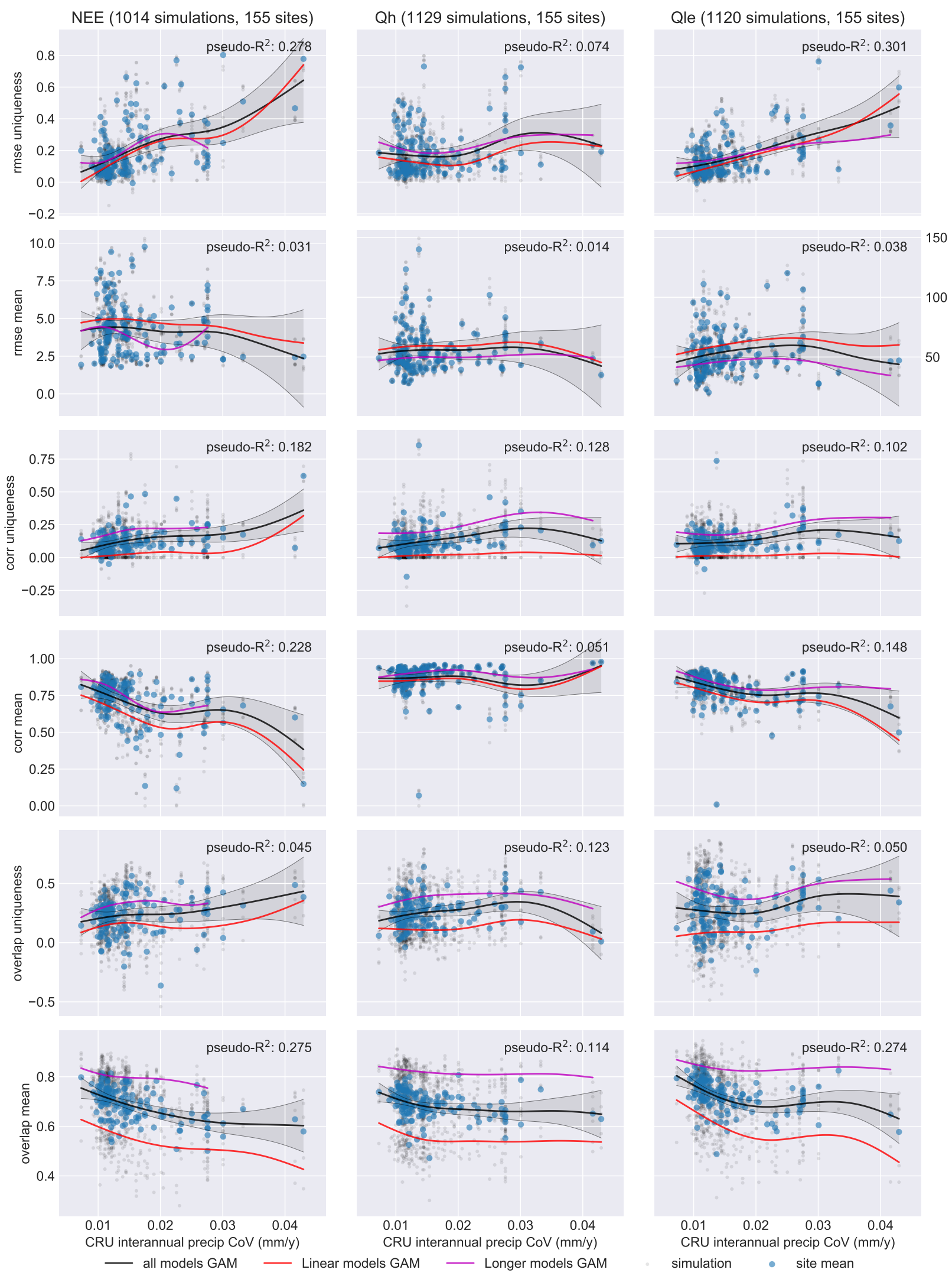

Figure 3: RMSE predictability by precipitation interannual variability, calculated from the coefficient of variation in the CRU TS4.01 annual means. 

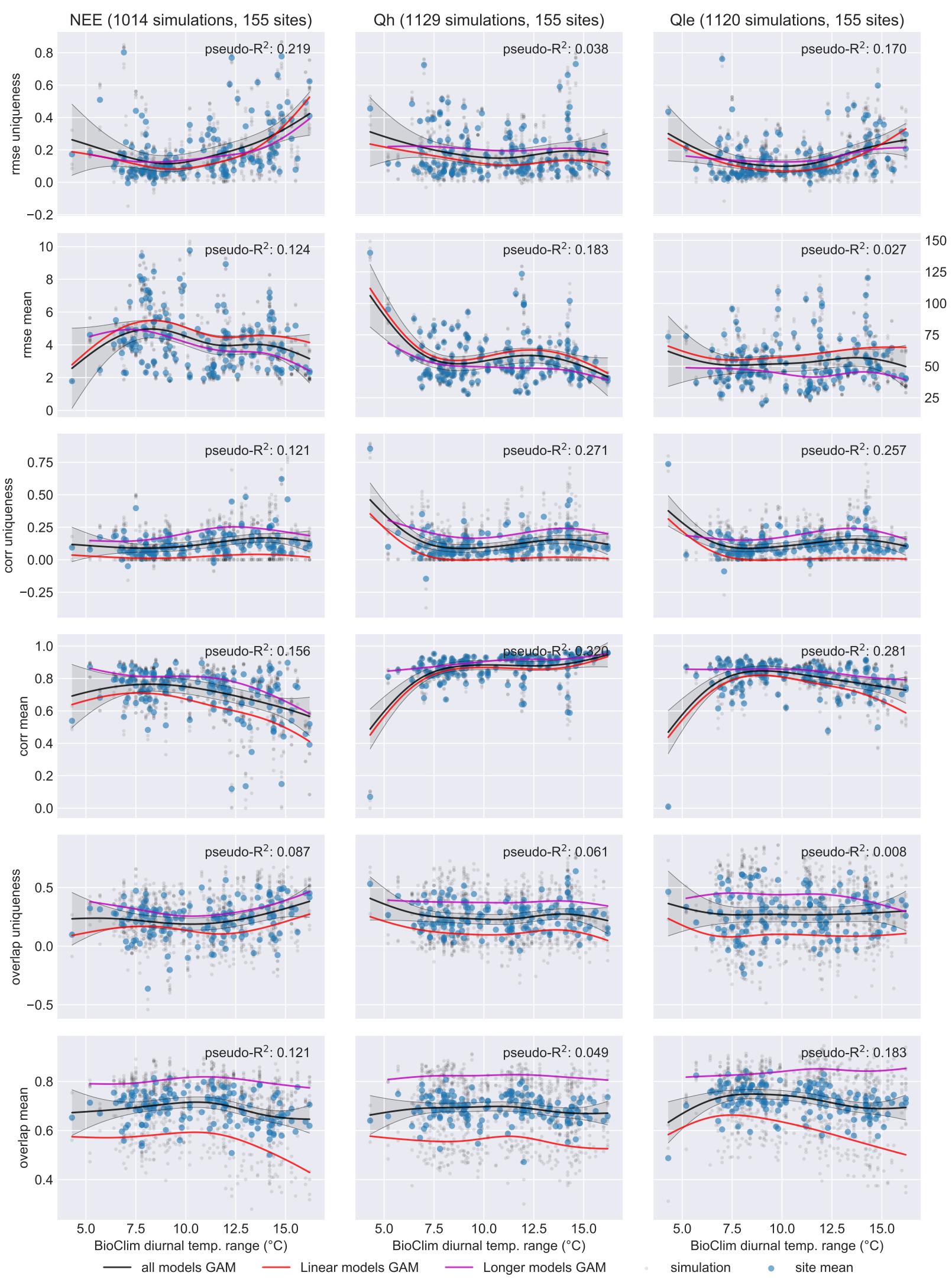

Figure 4: predictability metrics for temperature diurnal range. Note that higher diurnal temperature ranges are likely partially correlated with lower mean precipitation/humidity. 
${ }_{61}$ There is a hint of a trend towards higher uniqueness in sites that are driest in their wettest quarter, which is ${ }_{62}$ perhaps simply a reflection of the same effect seen in Figure 3 in the paper. Other determinants do not have 63 a clear patterns in predictability.

${ }_{64}$ There are some other patterns visible in some of the other predictability metrics, for example there appears 65 to be a trend towards a better overlap metric at sites with a higher BioClim_t_annual_range, as well as sites 66 with a higher BioClim_t_seasonality. 

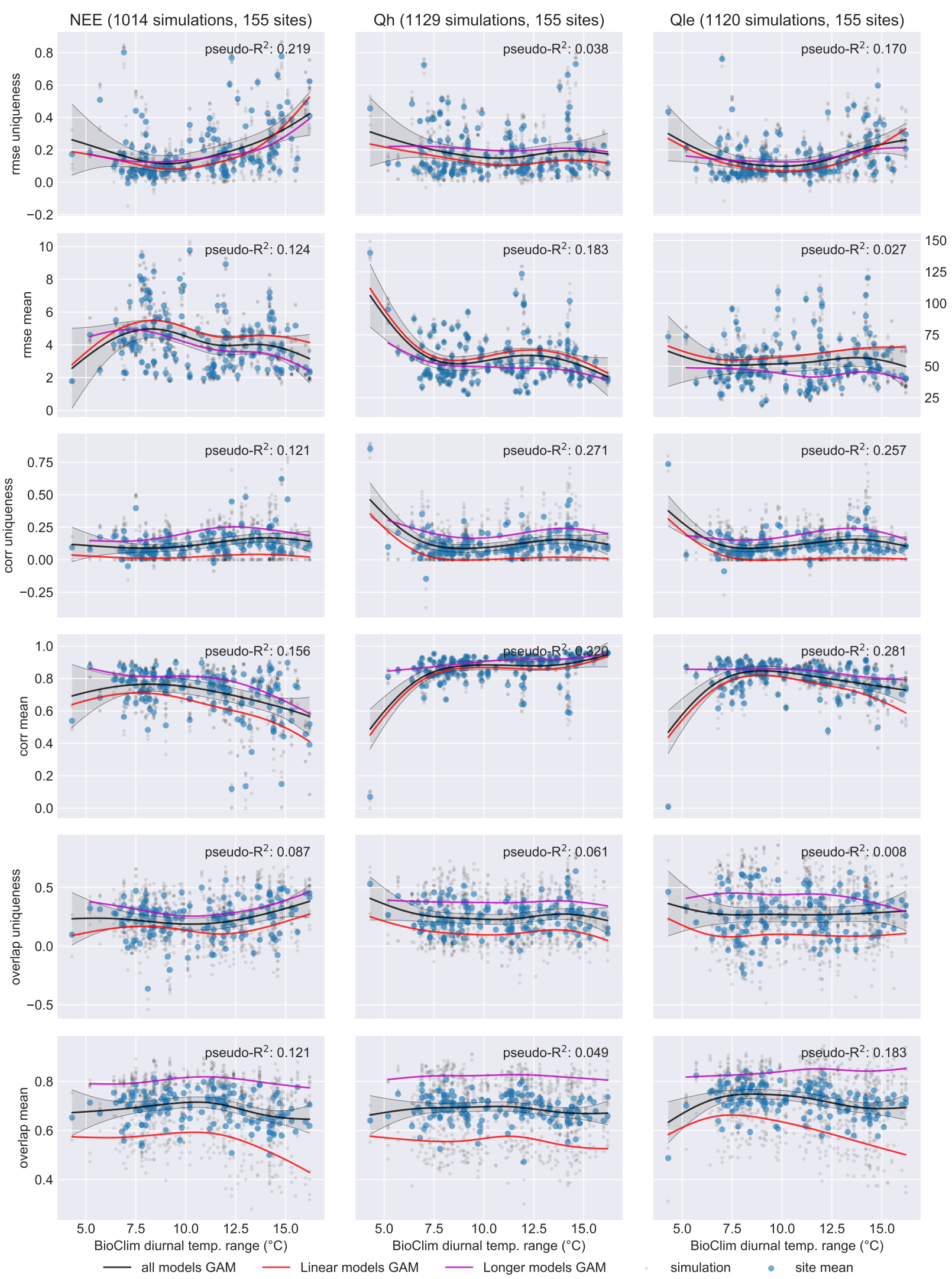

Figure 5: Predictability metrics for temperature diurnal range. Note: The first row is already included in the paper. 

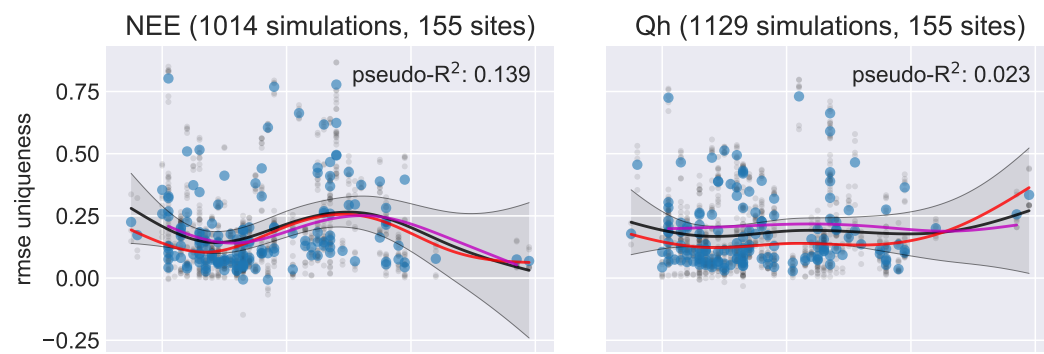

Qle (1120 simulations, 155 sites)
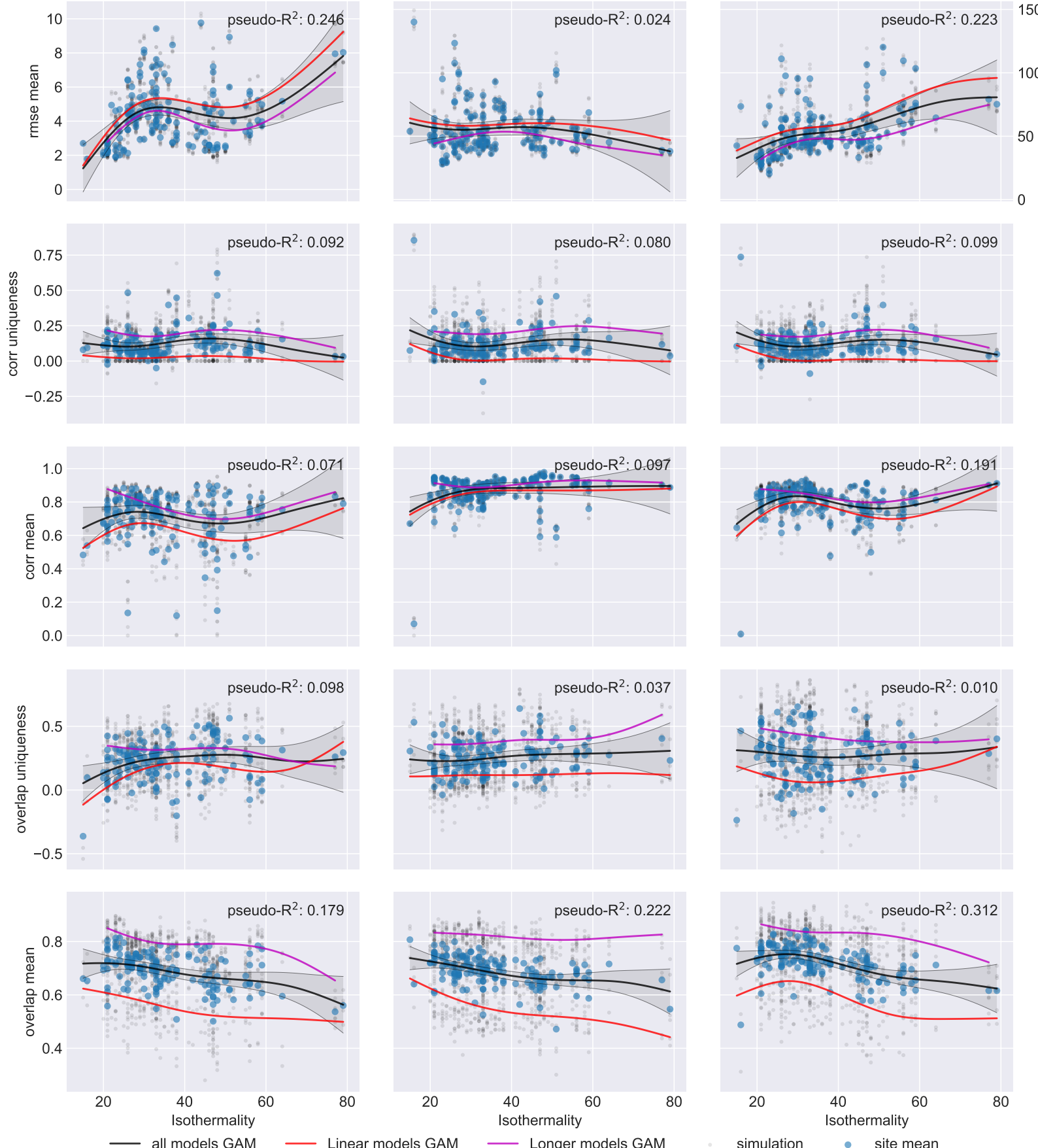

Figure 6: Predictability metrics for temperature isothermality. 

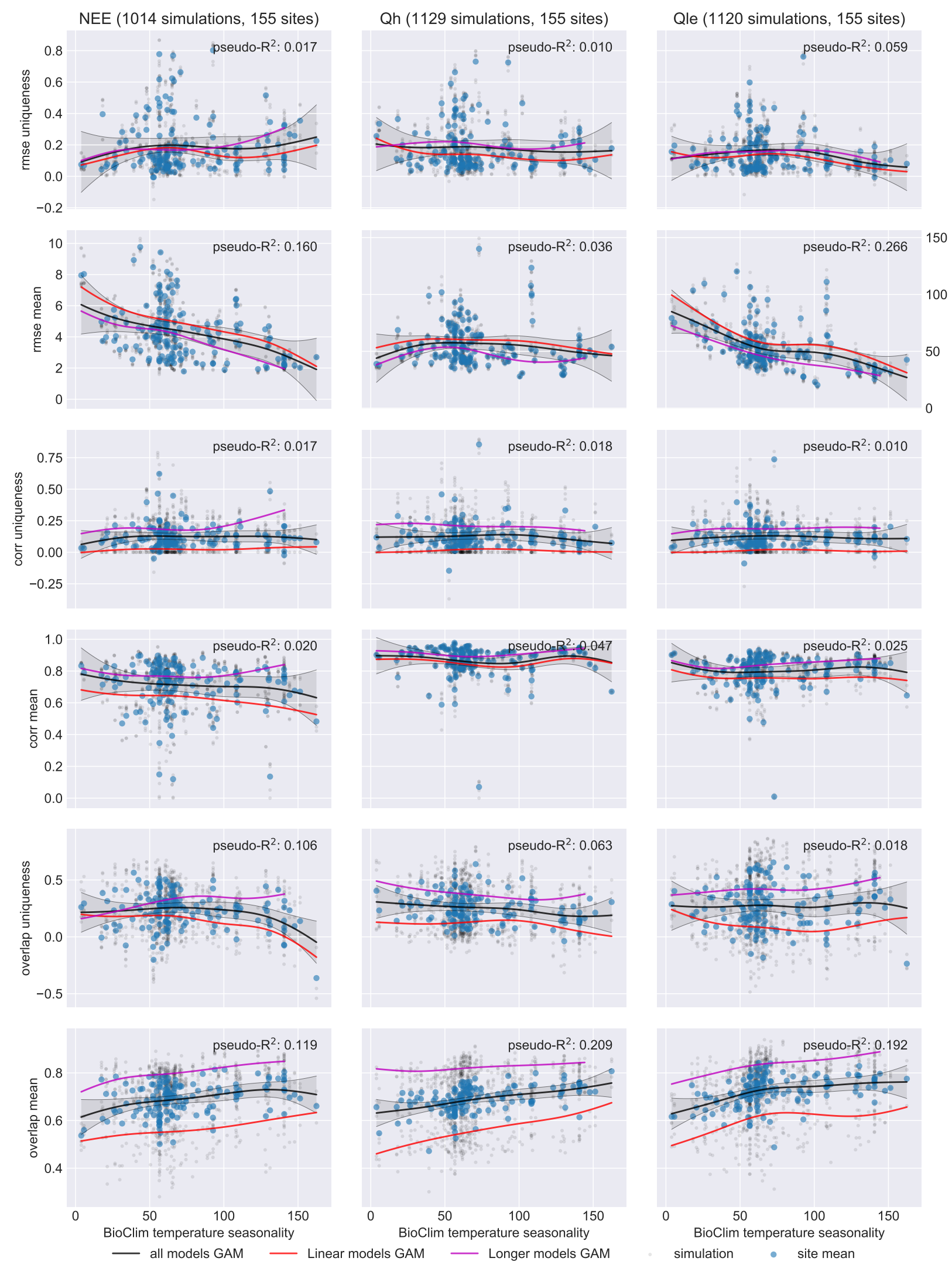

Figure 7: Predictability metrics for temperature seasonality. 

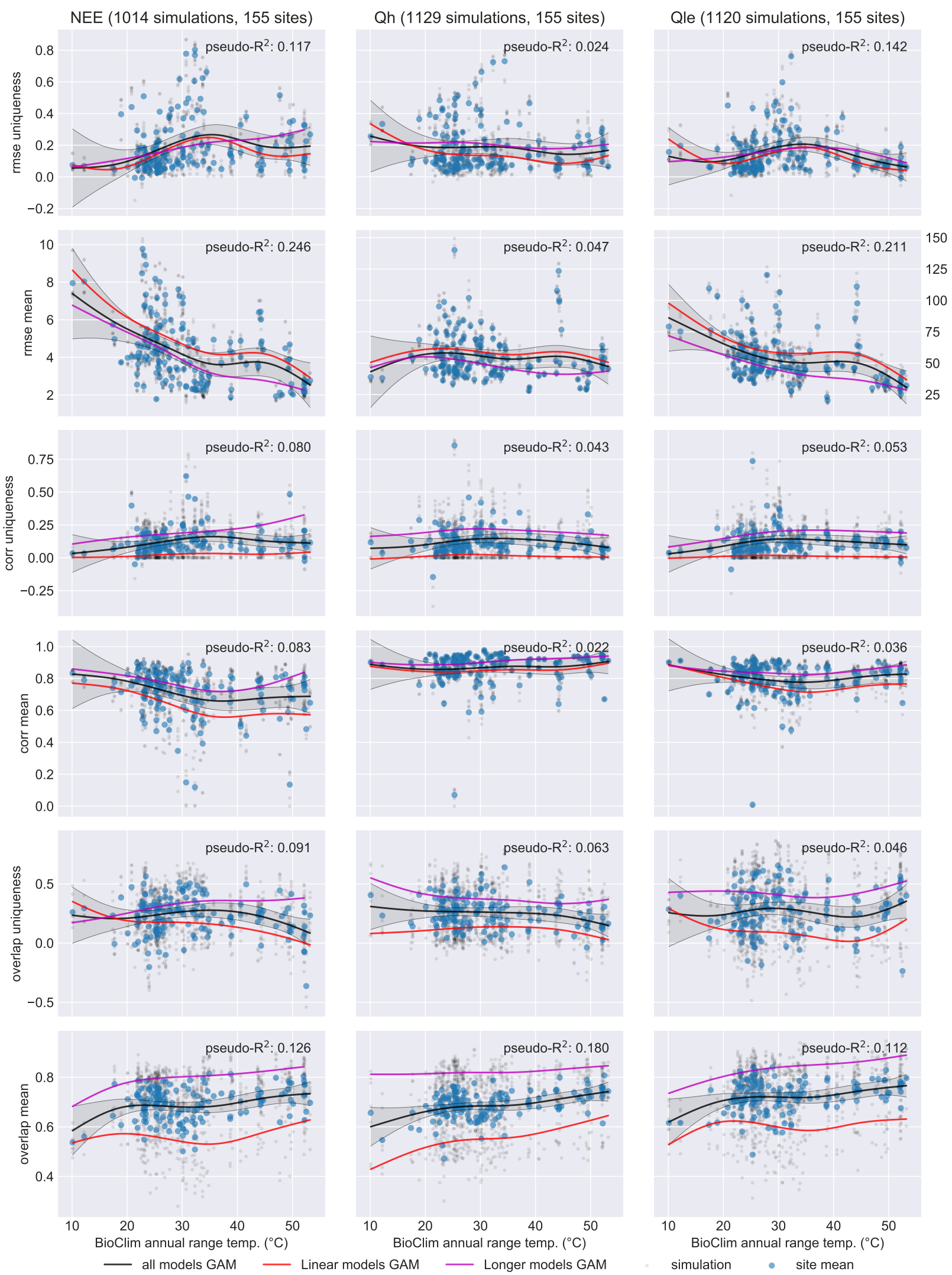

Figure 8: Predictability metrics for temperature annual range. 

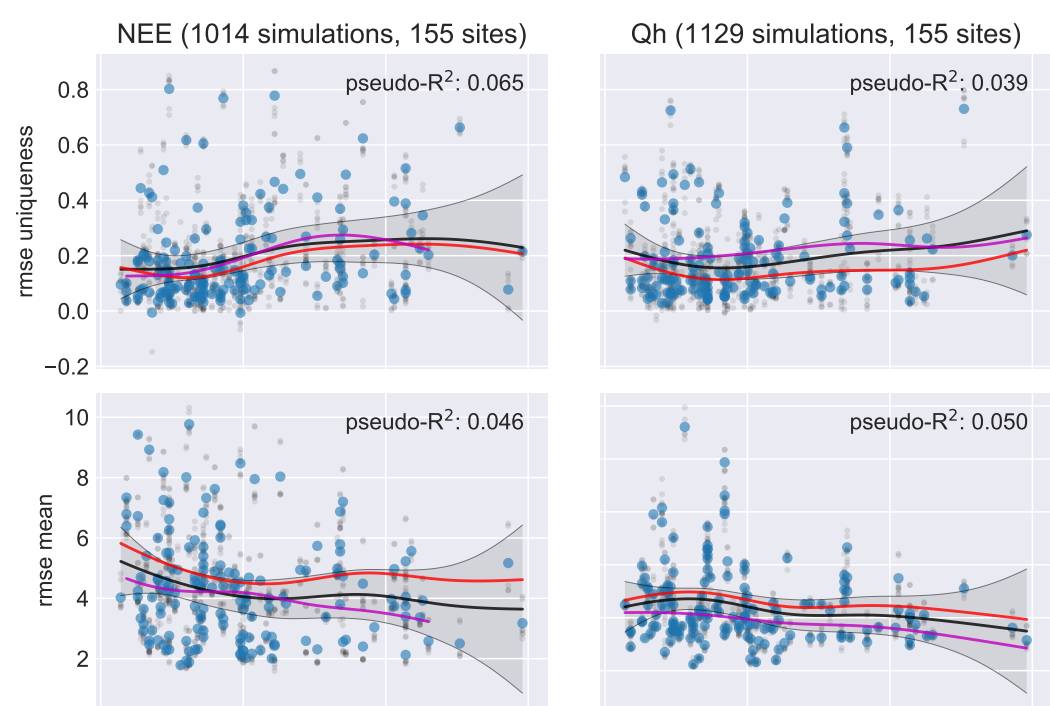

Qle (1120 simulations, 155 sites)
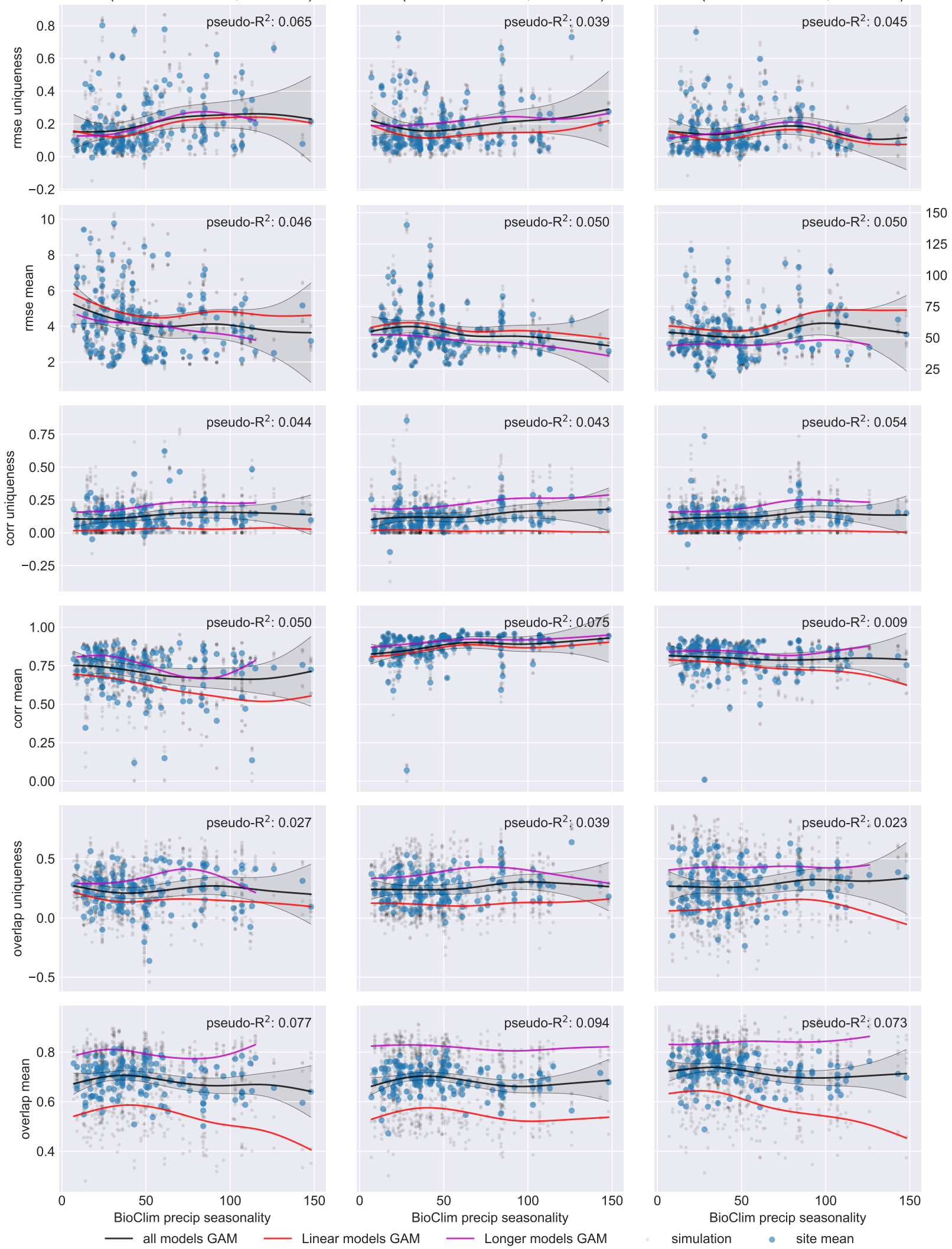

Figure 9: Predictability metrics for rainfall seasonality. 

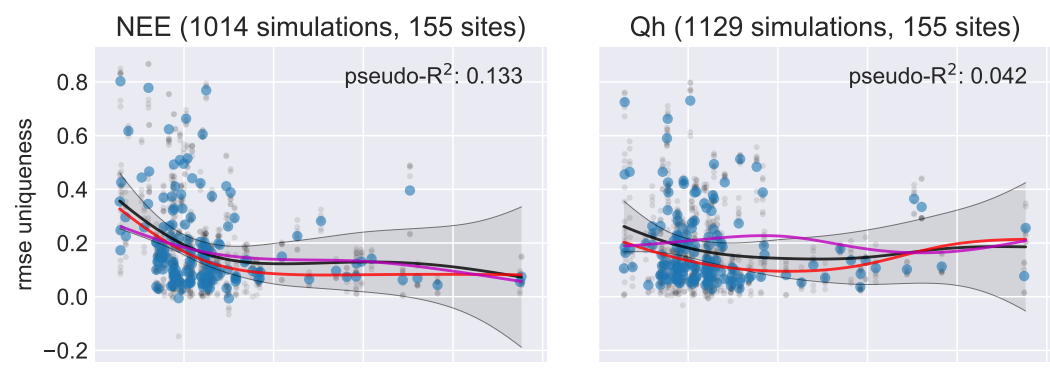

Qle (1120 simulations, 155 sites)
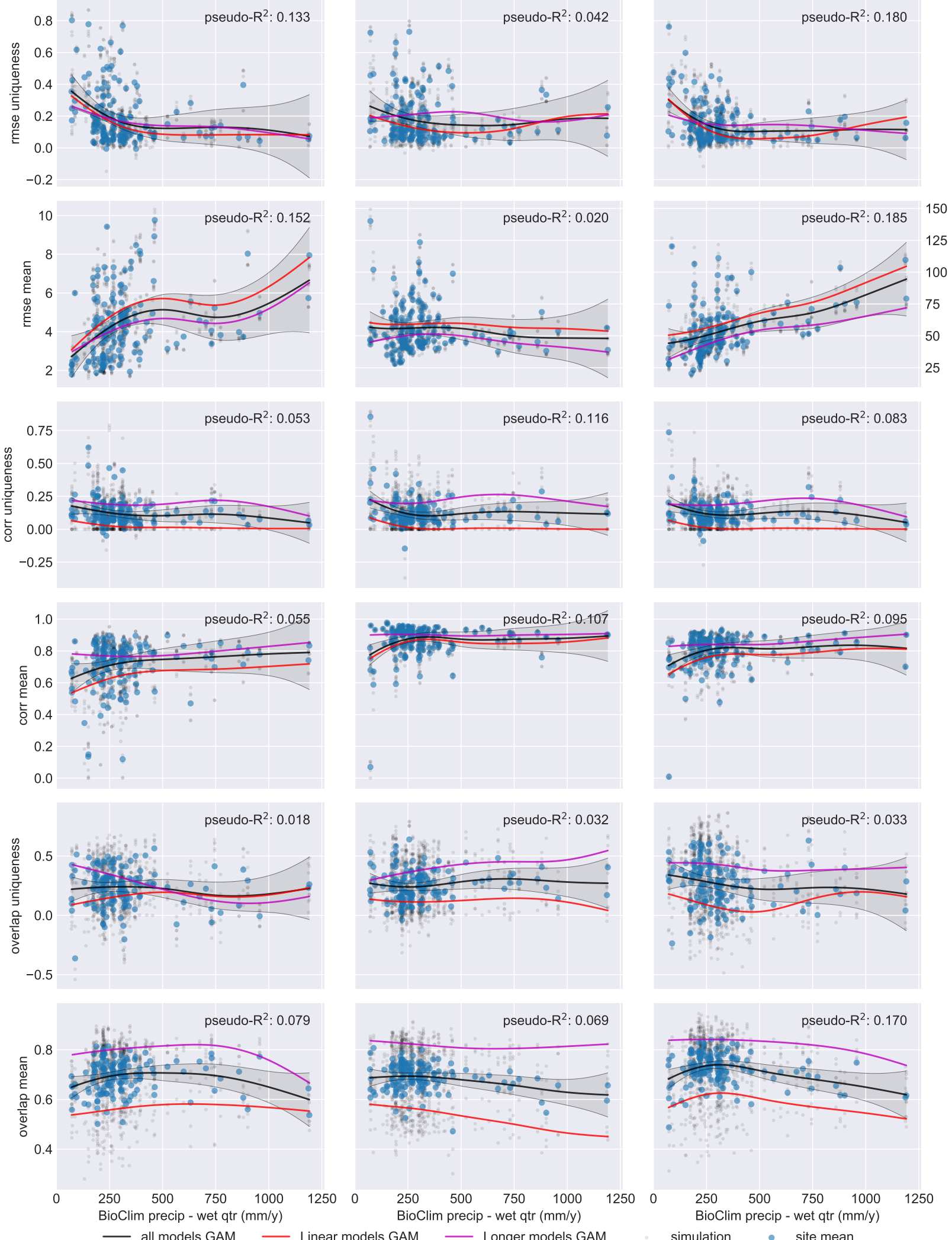

Figure 10: Predictability metrics for precipitation of the wettest quarter. 

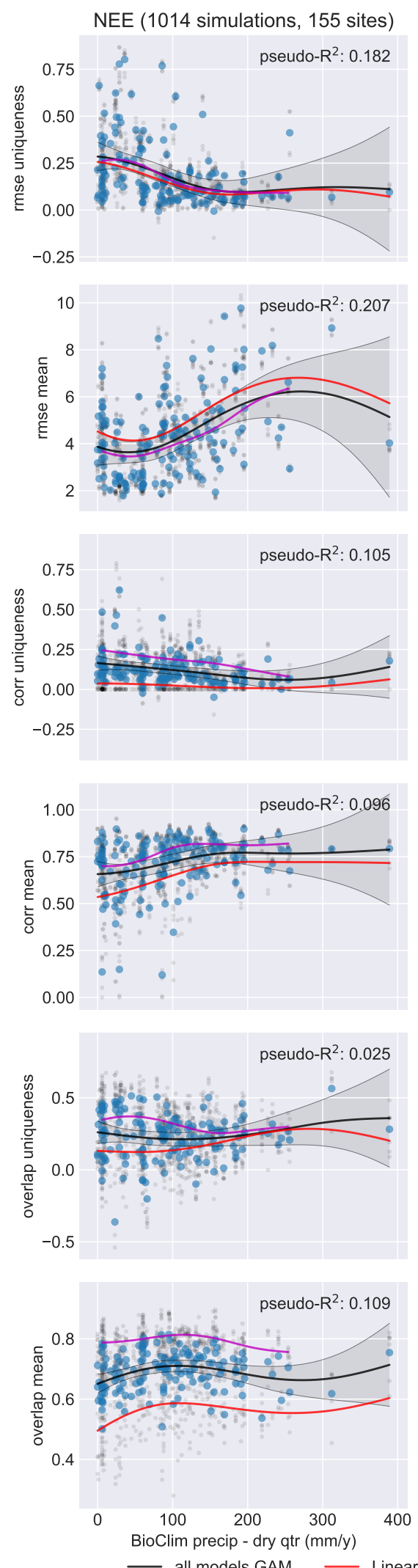
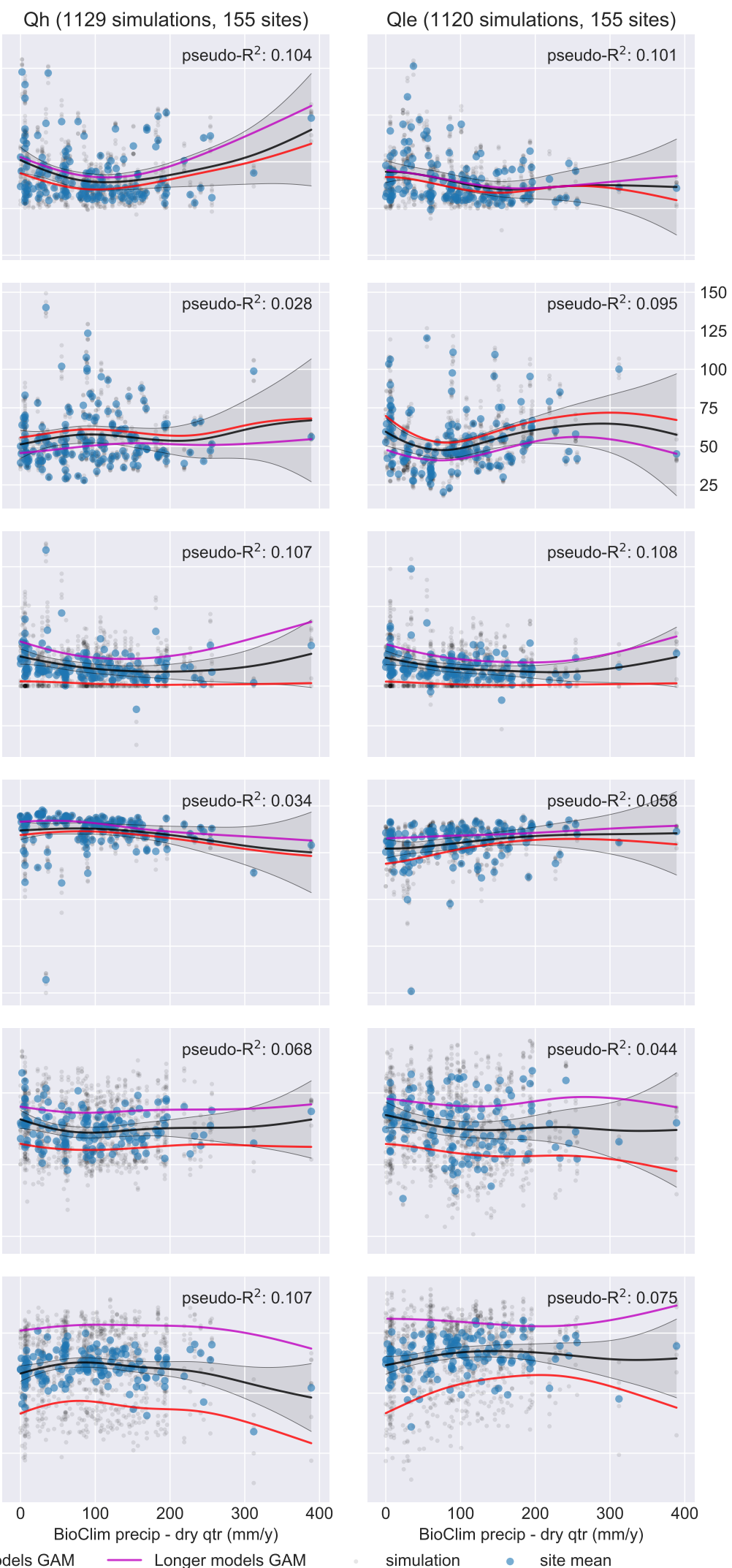

Figure 11: Predictability metrics for precipitation of the driest quarter. 


\section{${ }_{67}$ Extended Vegetation type analysis}

${ }_{68}$ This figure shows the other predictability metrics for grouped vegetation type which were omitted from the 69 paper. 

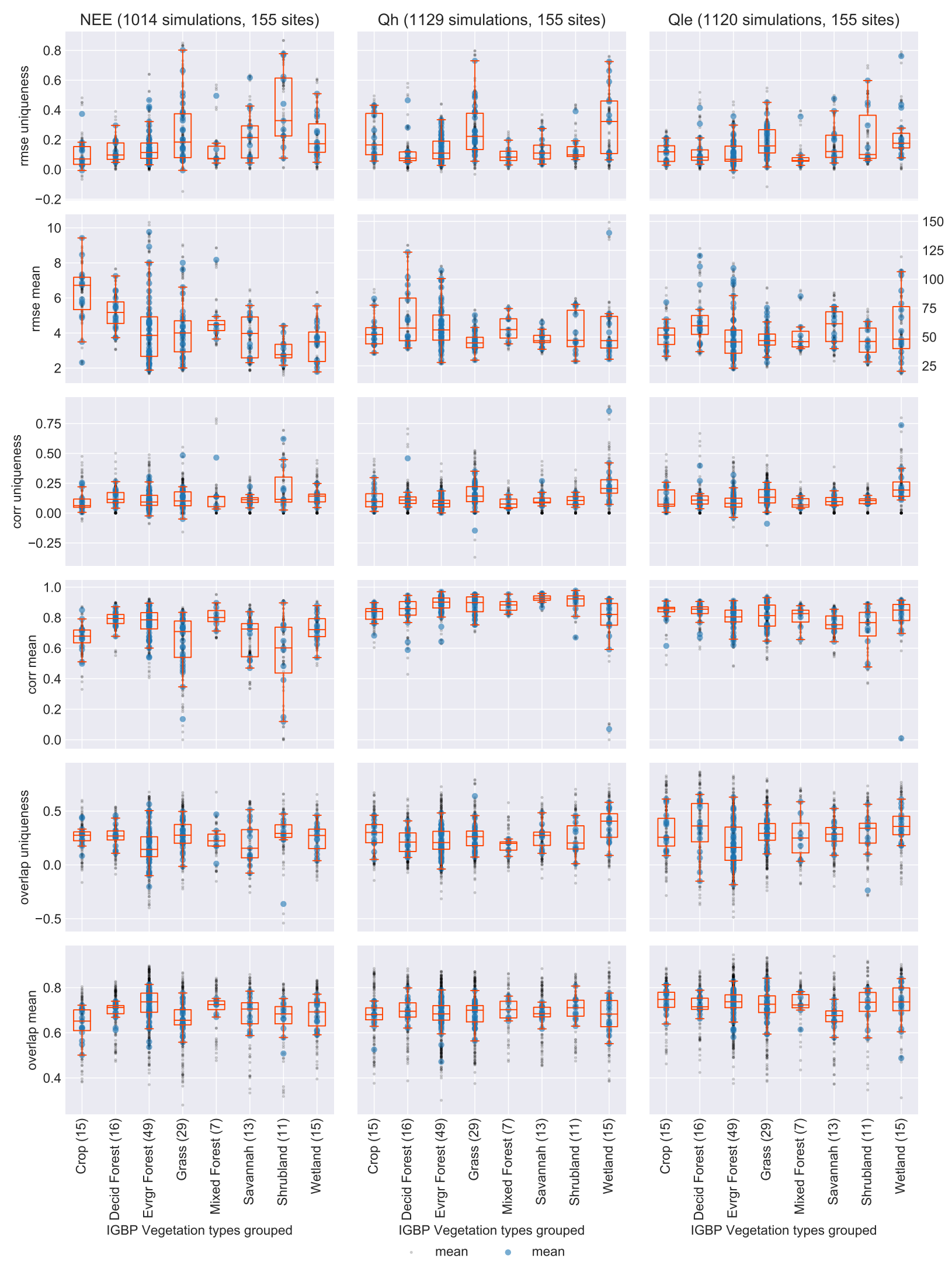

Figure 12: Predictability metrics for vegetation type (grouped, see Methods). 


\section{Geographic analysis}

We are training the global models on all available sites, but FLUXNET sites are not evenly distributed over the land area of the globe. As such, we might expect that sites that have many other similar sites in the global dataset would have their behaviours more adequately captured by a globally trained model. To investigate whether more geographically unique sites were less predictable, we mapped the sites by uniqueness, and also compared uniqueness by average distance to all other sites.

\section{Results}

This section includes maps of RMSE uniqueness mean for Qh and Qle, mapped as per Figure 11 in the paper, as well as the remoteness predictability metrics. Distribution of uniqueness appears to be different for Qh (more high-uniqueness sites), but over-all, both variables have a similar, but lest distinct pattern of uniqueness as seen in NEE in Figure 11.

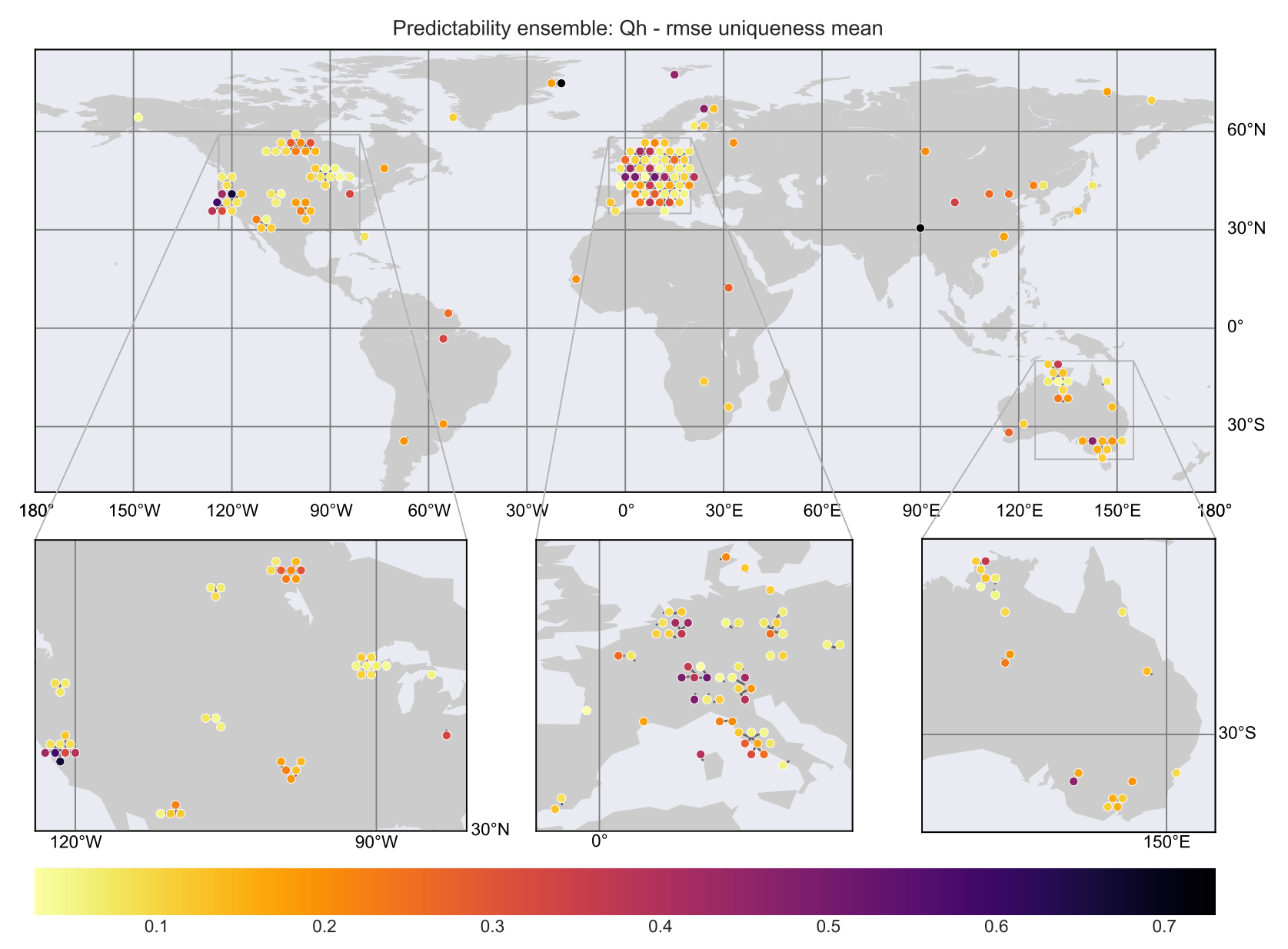

Figure 13: Map of Qh predictability - RMSE uniqueness, averaged across models, as per Figure 11 in the paper. 


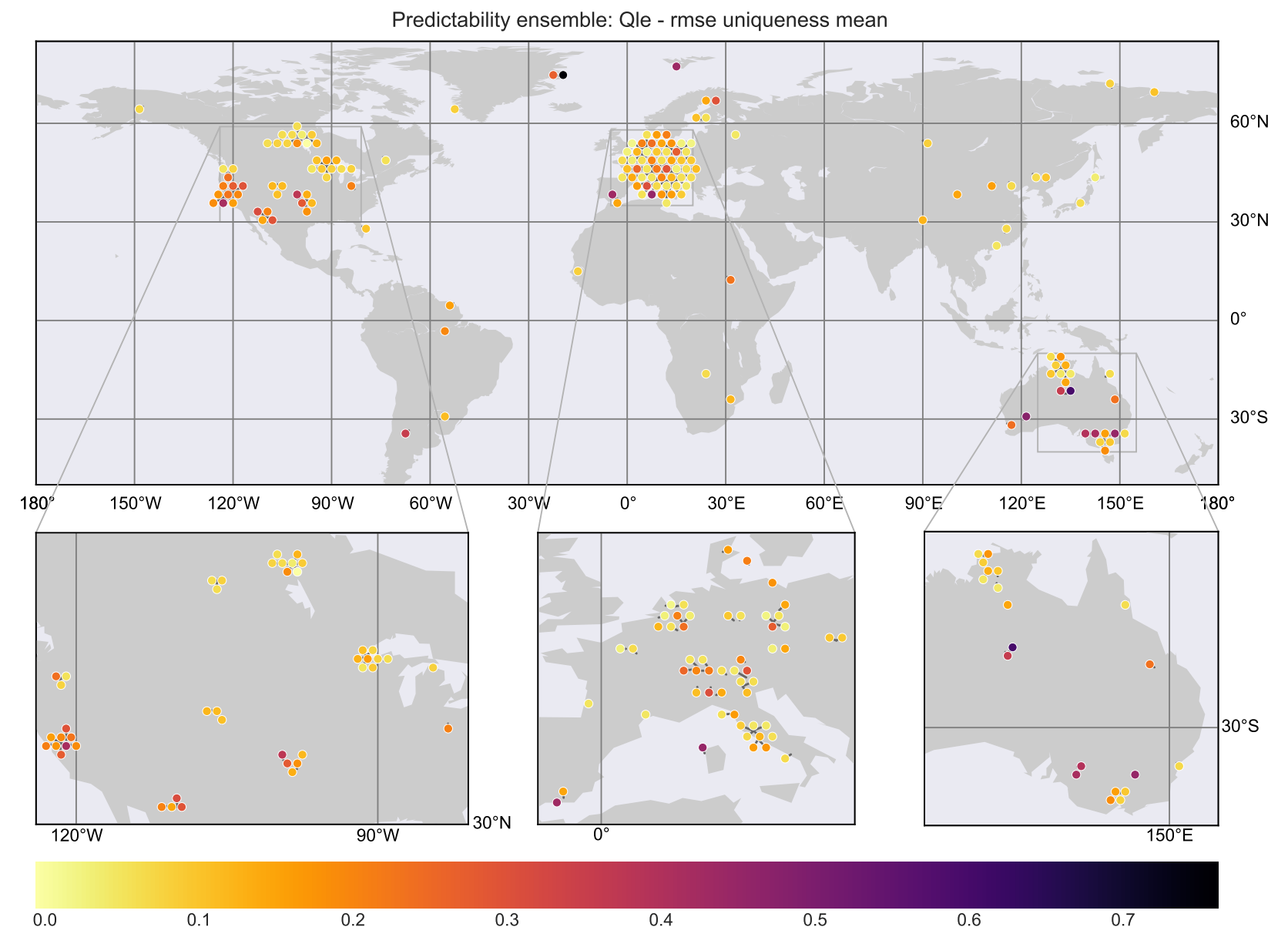

Figure 14: Map of Qle predictability - RMSE uniqueness, averaged across models, as per Figure 11 in the paper. 

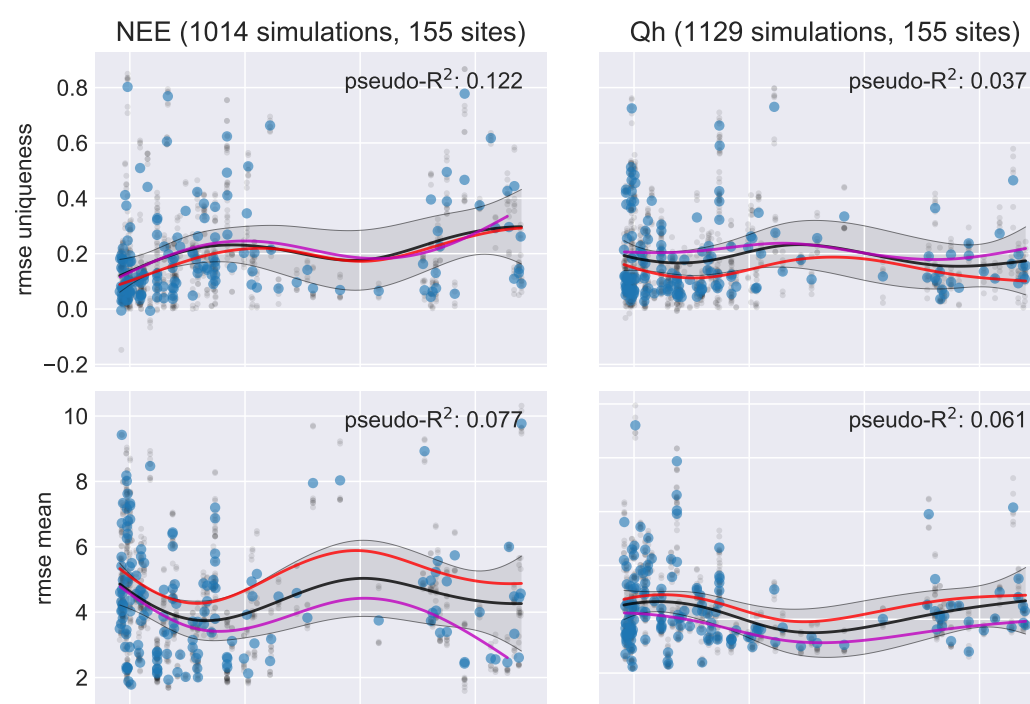

Qle (1120 simulations, 155 sites)
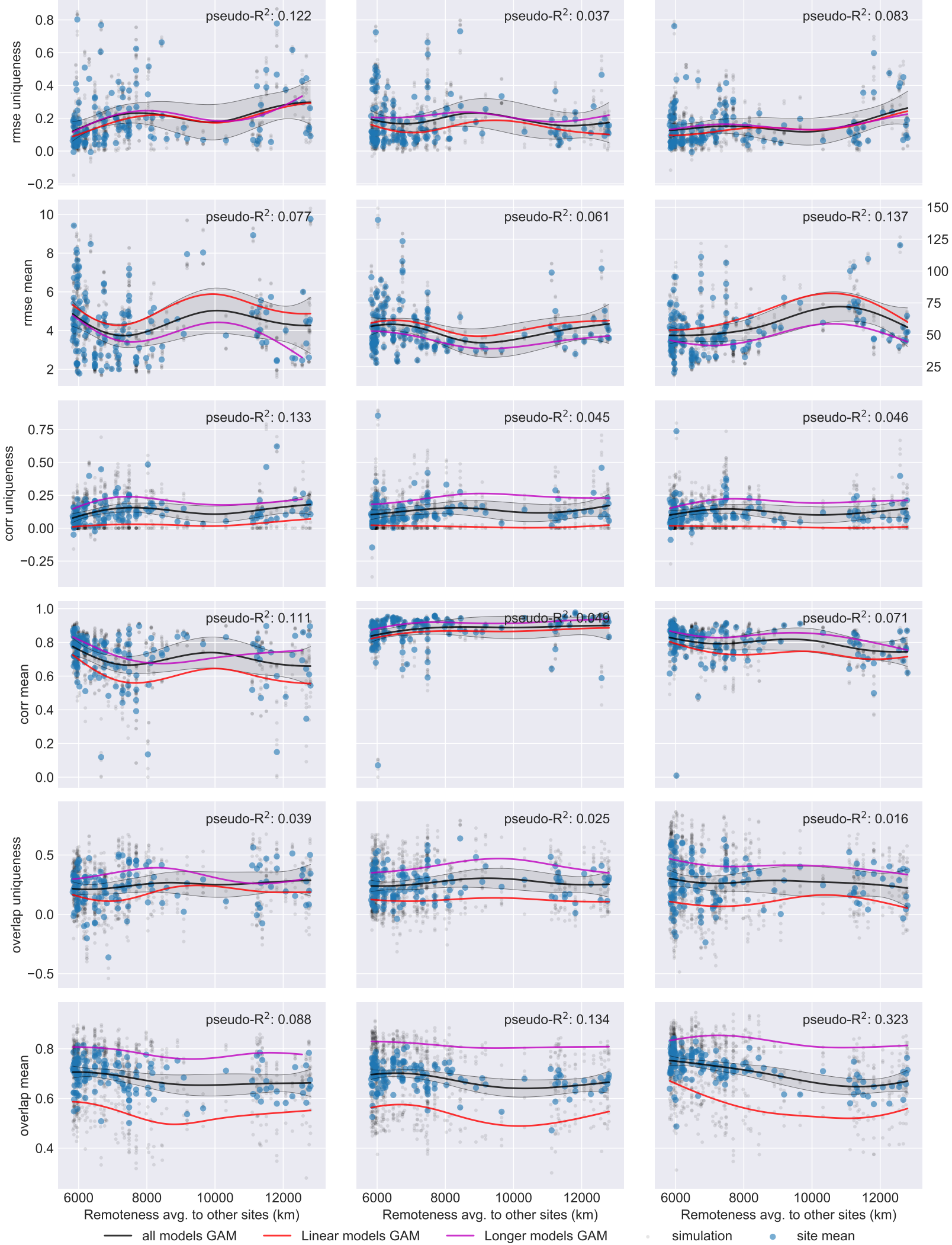

Figure 15: Predictability metrics by remoteness (average distance to all other sites) 

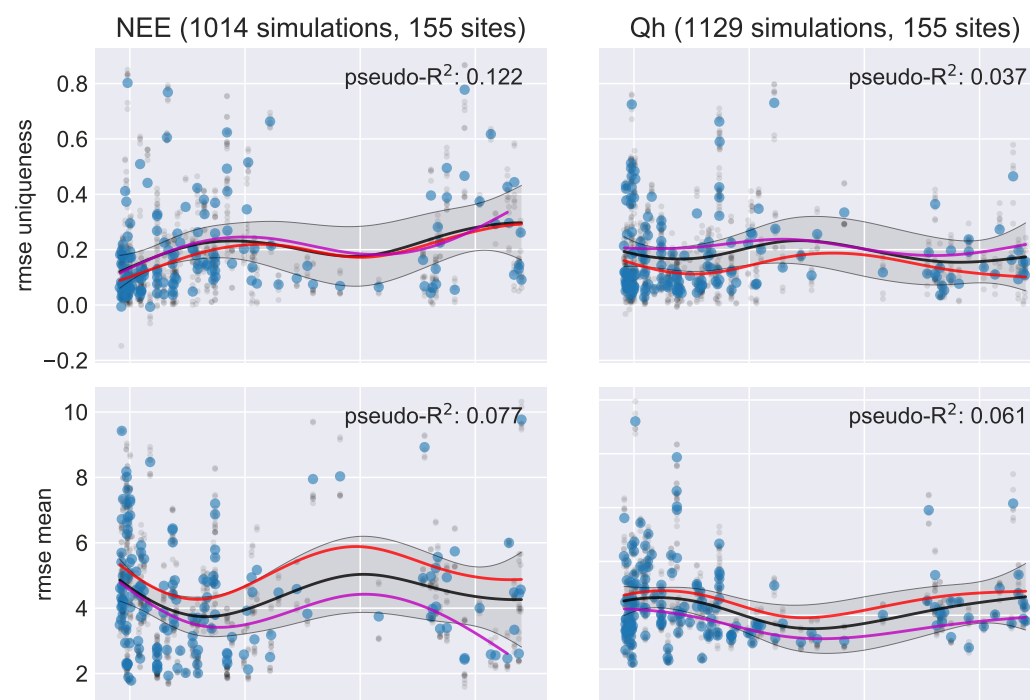

Qle (1120 simulations, 155 sites)
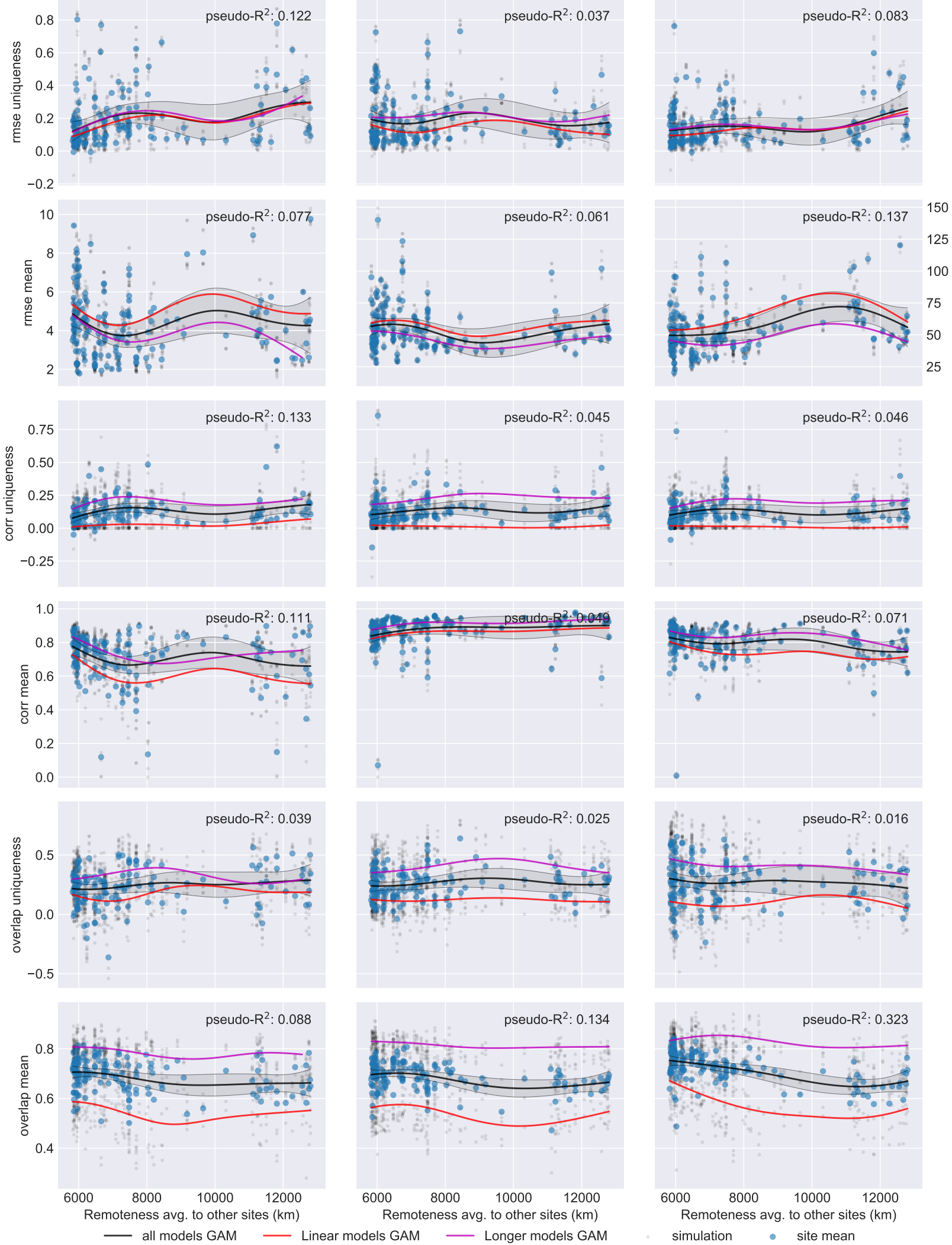

Predictability metrics by average remoteness 


\section{${ }_{81}$ Energy Gap Closure analysis}

${ }_{82}$ The energy closure problem in FLUXNET is investigated in Figure 16, where we show the actual gap (in

83 $\left.\mathrm{W} / \mathrm{m}^{2}\right)$, and in Figure 17 where we show the absolute energy gap normalised by Rnet. In the first figure,

84 there is no trend in any flux. In the second figure, there is appears to be a tend toward higher uncertainty in

85 sites with large energy gaps relative to their total Rnet, however this trend is quite uncertain, due to the low

86 number of sites involved. 

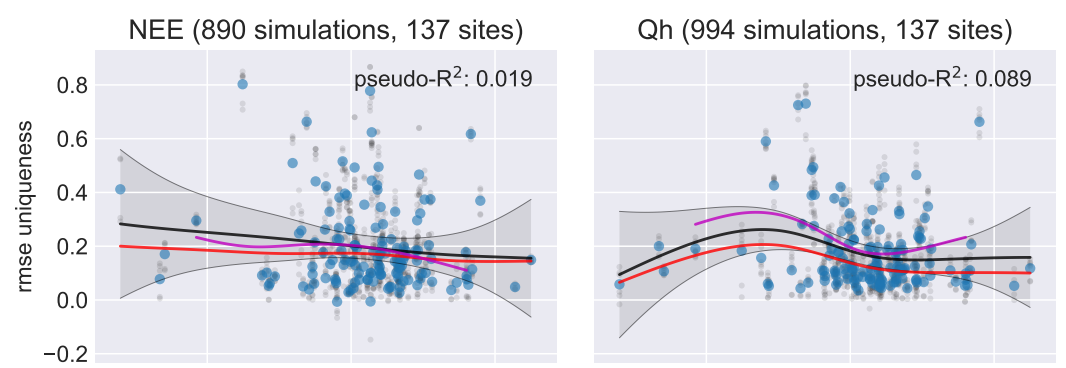

Qle (985 simulations, 137 sites)
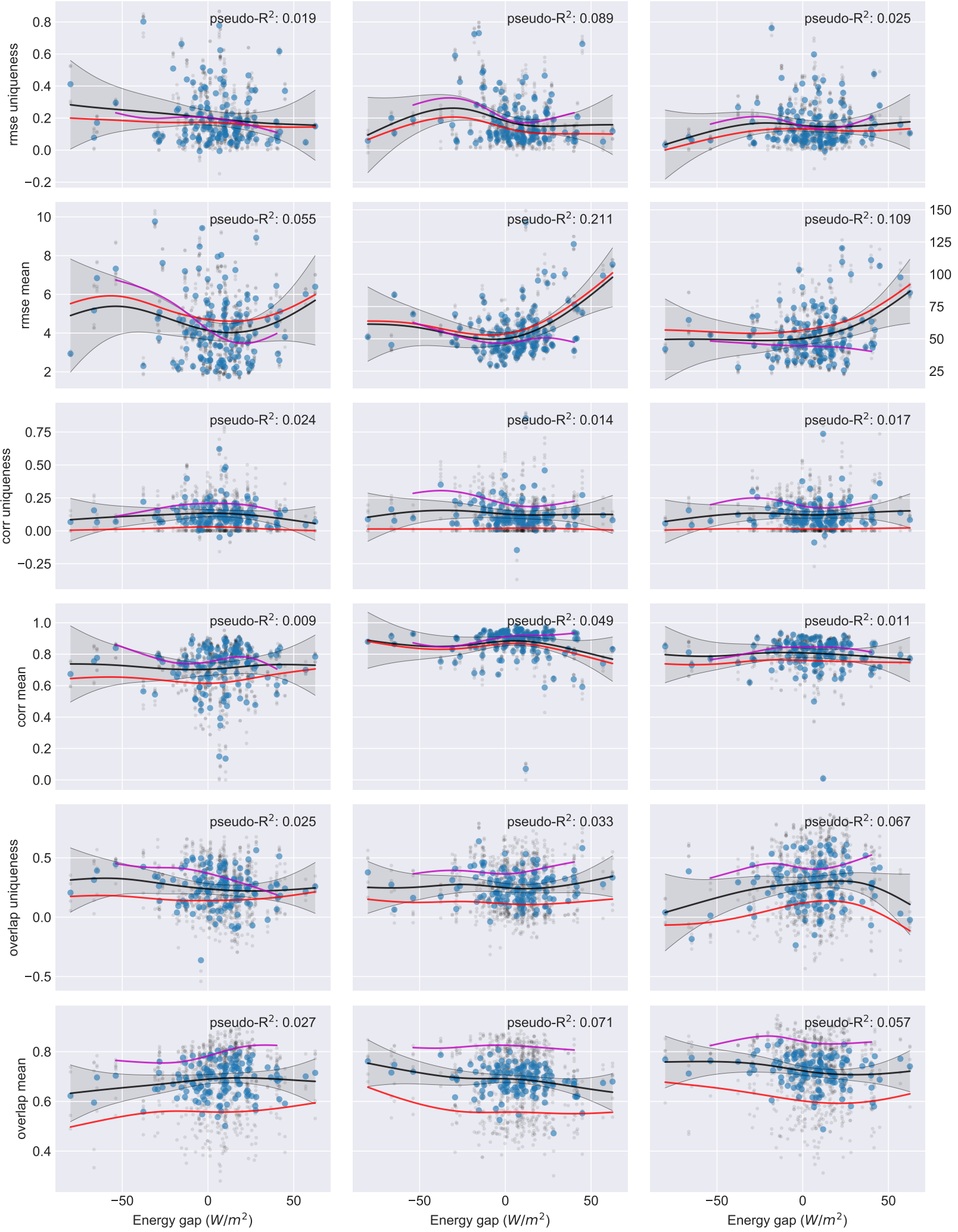

— all models GAM
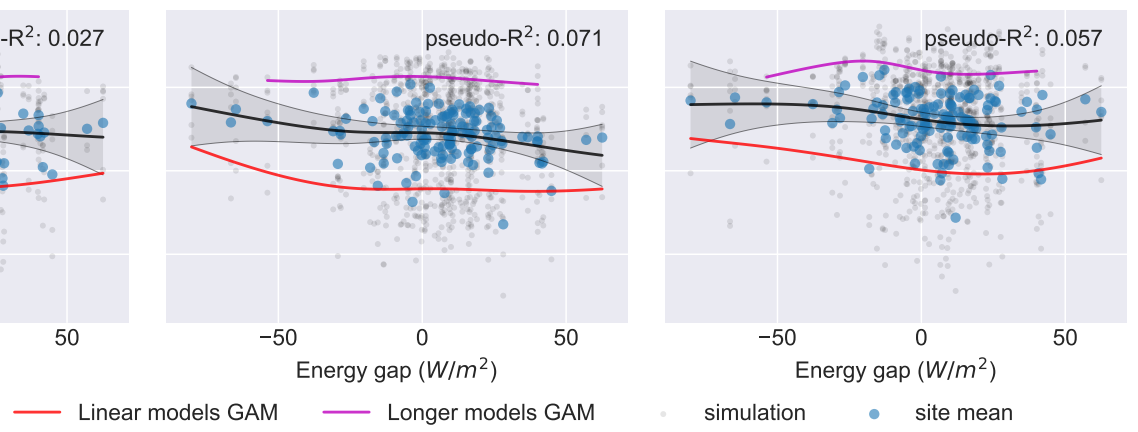

Figure 16: Predictability metrics for energy gap $\left(W / m^{2}\right)$. Sites wth positive energy gaps have too much Rnet to relative to the over heat fluxes. 

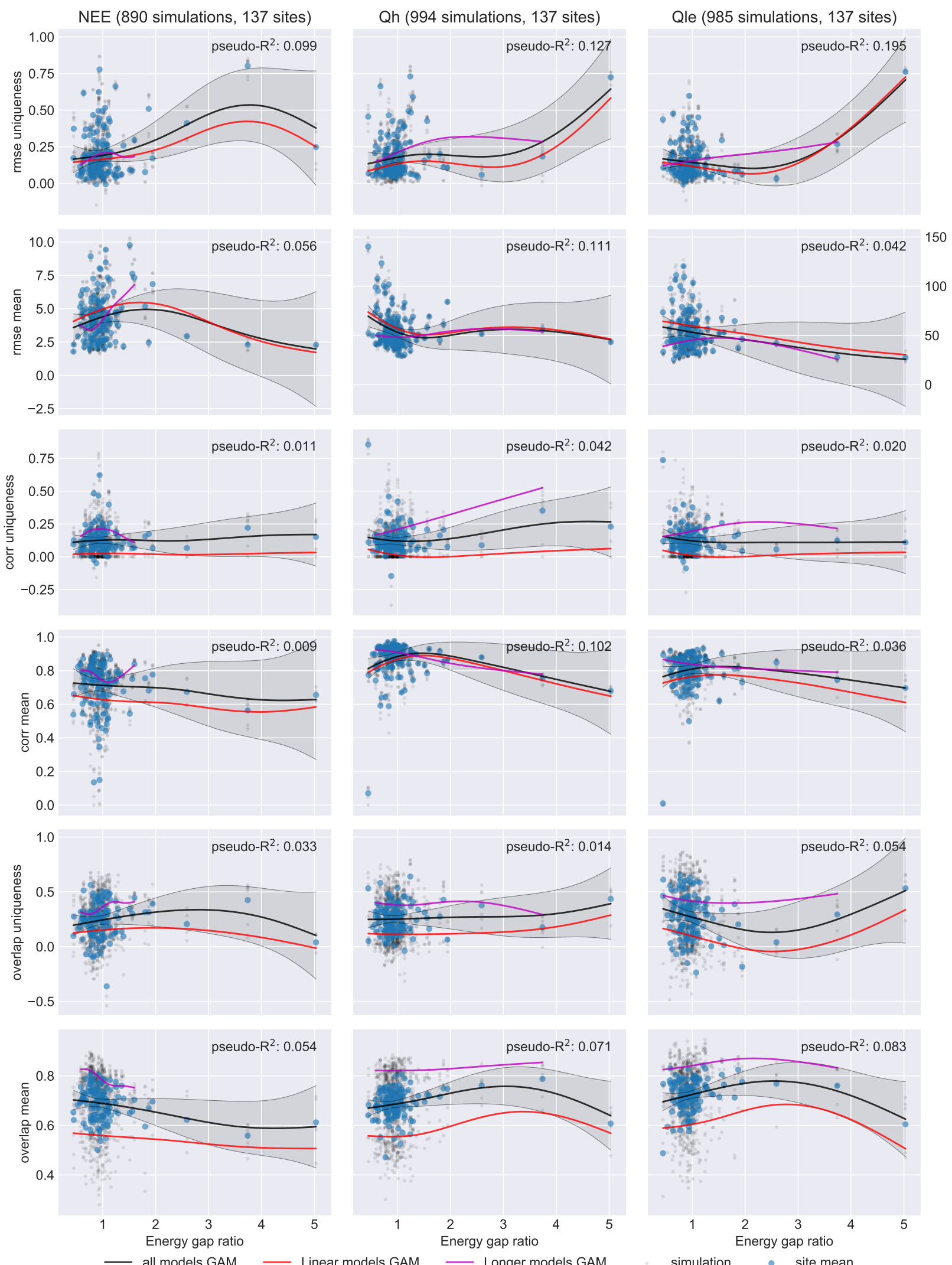

Figure 17: Predictability metrics for energy gap normalised by Rnet. 


\section{${ }_{87}$ Extended dataset length analysis}

${ }_{88}$ The following plot shows the predictability metrics by data set length that were omitted from Figure 13. 

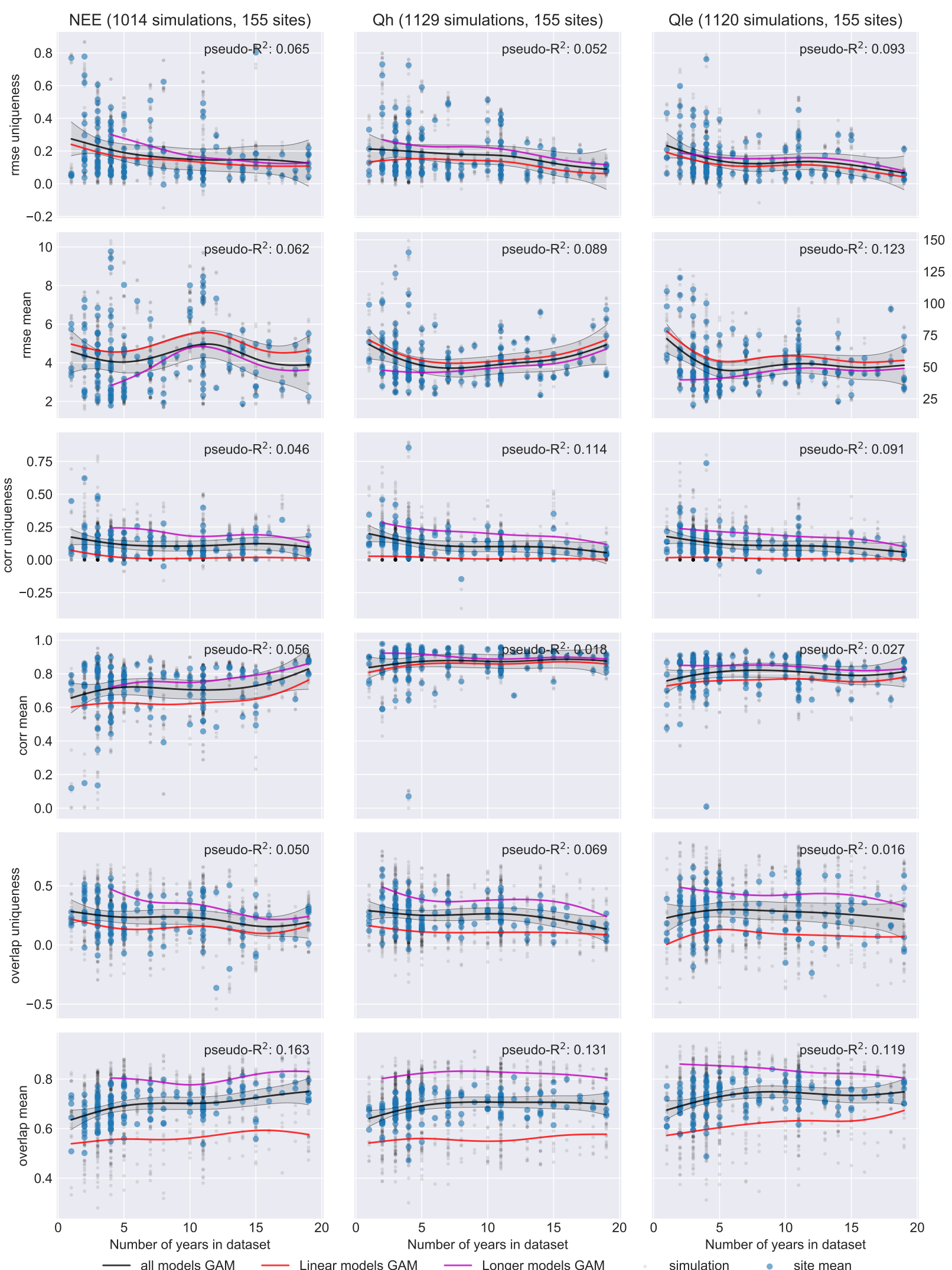

Predictability metrics for number of years in dataset. 


\section{Fluxnet Citations}

Sites, vegetation types, locations and studied periods of flux sites used in this analysis. All data originally from www.fluxdata.org, via https://github.com/trevorkeenan/FLUXNET_citations. Vegetation types: deciduous broadleaf forest (DBF); evergreen broadleaf forest (EBF); evergreen needleleaf forest (ENF); grassland (GRA); mixed deciduous and evergreen needleleaf forest (MF); savanna ecosystem (SAV); shrub ecosystem (SHR); wetland (WET).

\begin{tabular}{|c|c|c|c|c|c|}
\hline Site code & Veg type & Latitude & Longitude & Period & References \\
\hline AR-SLu & $\mathrm{MF}$ & -33.4648 & -66.4598 & 2009-2011 & (Ulke et al., 2015) \\
\hline AR-Vir & ENF & -28.2395 & -56.1886 & 2009-2012 & (Posse et al., 2016) \\
\hline AT-Neu & GRA & 47.1167 & 11.3175 & 2002-2012 & (Wohlfahrt et al., 2008) \\
\hline AU-Ade & WSA & -13.0769 & 131.1178 & 2007-2009 & (Beringer et al., 2011c) \\
\hline AU-ASM & ENF & -22.2830 & 133.2490 & 2010-2013 & (Cleverly et al., 2013) \\
\hline AU-Cpr & SAV & -34.0021 & 140.5891 & 2010-2014 & (Meyer et al., 2015) \\
\hline AU-Cum & $\mathrm{EBF}$ & -33.6133 & 150.7225 & 2012-2014 & (Beringer et al., 2016a) \\
\hline AU-DaP & GRA & -14.0633 & 131.3181 & $2007-2013$ & (Beringer et al., 2011a) \\
\hline AU-DaS & SAV & -14.1593 & 131.3881 & 2008-2014 & (Hutley et al., 2011) \\
\hline AU-Dry & $\mathrm{SAV}$ & -15.2588 & 132.3706 & $2008-2014$ & (Cernusak et al., 2011) \\
\hline AU-Emr & GRA & -23.8587 & 148.4746 & 2011-2013 & (Schroder et al., 2014) \\
\hline AU-Fog & WET & -12.5452 & 131.3072 & 2006-2008 & (Beringer et al., 2013) \\
\hline AU-Gin & WSA & -31.3764 & 115.7138 & 2011-2014 & (Beringer et al., 2016d) \\
\hline AU-GWW & SAV & -30.1913 & 120.6541 & 2013-2014 & (Prober et al., 2012) \\
\hline AU-How & WSA & -12.4943 & 131.1523 & 2001-2014 & (Cernusak, 2007) \\
\hline AU-Lox & DBF & -34.4704 & 140.6551 & 2008-2009 & (Stevens et al., 2011) \\
\hline AU-RDF & WSA & -14.5636 & 132.4776 & 2011-2013 & (Bristow et al., 2016) \\
\hline AU-Rig & GRA & -36.6499 & 145.5759 & 2011-2014 & (Beringer et al., 2016b) \\
\hline AU-Rob & $\mathrm{EBF}$ & -17.1175 & 145.6301 & 2014-2014 & (Beringer et al., 2016c) \\
\hline AU-Stp & GRA & -17.1507 & 133.3502 & $2008-2014$ & (Beringer et al., 2011b) \\
\hline AU-TTE & $\mathrm{OSH}$ & -22.2870 & 133.6400 & 2012-2013 & (Cleverly et al., 2016) \\
\hline AU-Wac & $\mathrm{EBF}$ & -37.4259 & 145.1878 & $2005-2008$ & (Kilinc et al., 2013) \\
\hline AU-Whr & $\mathrm{EBF}$ & -36.6732 & 145.0294 & 2011-2014 & (McHugh et al., 2017) \\
\hline AU-Wom & $\mathrm{EBF}$ & -37.4222 & 144.0944 & 2010-2012 & (Hinko-Najera et al., 2017) \\
\hline AU-Ync & GRA & -34.9893 & 146.2907 & $2012-2014$ & (Yee et al., 2015) \\
\hline BE-Bra & $\mathrm{MF}$ & 51.3092 & 4.5206 & 1996-2014 & (Carrara et al., 2004) \\
\hline BE-Lon & $\mathrm{CRO}$ & 50.5516 & 4.7461 & 2004-2014 & (Moureaux et al., 2006) \\
\hline BE-Vie & $\mathrm{MF}$ & 50.3051 & 5.9981 & 1996-2014 & (Aubinet et al., 2001) \\
\hline BR-Sa3 & $\mathrm{EBF}$ & -3.0180 & -54.9714 & $2000-2004$ & (Wick et al., 2005) \\
\hline CA-Man & ENF & 55.8796 & -98.4808 & $1994-2008$ & (Dunn et al., 2007) \\
\hline CA-NS1 & ENF & 55.8792 & -98.4839 & 2001-2005 & (Goulden et al., 2006a) \\
\hline CA-NS2 & ENF & 55.9058 & -98.5247 & $2001-2005$ & (Goulden et al., 2006b) \\
\hline CA-NS3 & ENF & 55.9117 & -98.3822 & 2001-2005 & (Goulden et al., 2006c) \\
\hline $\mathrm{CA}-\mathrm{NS} 4$ & ENF & 55.9144 & -98.3806 & $2002-2005$ & (Goulden et al., 2006d) \\
\hline CA-NS5 & ENF & 55.8631 & -98.4850 & 2001-2005 & (Goulden et al., 2006e) \\
\hline CA-NS6 & $\mathrm{OSH}$ & 55.9167 & -98.9644 & 2001-2005 & (Goulden et al., 2006f) \\
\hline CA-NS7 & $\mathrm{OSH}$ & 56.6358 & -99.9483 & $2002-2005$ & (Goulden et al., 2006g) \\
\hline CA-Qfo & ENF & 49.6925 & -74.3421 & 2003-2010 & (Bergeron et al., 2007) \\
\hline $\mathrm{CA}-\mathrm{SF} 1$ & ENF & 54.4850 & -105.8176 & $2003-2006$ & (Mkhabela et al., 2009a) \\
\hline CA-SF2 & ENF & 54.2539 & -105.8775 & 2001-2005 & (Mkhabela et al., 2009b) \\
\hline CA-SF3 & $\mathrm{OSH}$ & 54.0916 & -106.0053 & 2001-2006 & (Mkhabela et al., 2009c) \\
\hline CH-Cha & GRA & 47.2102 & 8.4104 & $2005-2014$ & (Merbold et al., 2014) \\
\hline CH-Dav & ENF & 46.8153 & 9.8559 & $1997-2014$ & (Zielis et al., 2014) \\
\hline
\end{tabular}




\begin{tabular}{|c|c|c|c|c|c|}
\hline Site code & Veg type & Latitude & Longitude & Period & References \\
\hline $\mathrm{CH}-\mathrm{Fru}$ & GRA & 47.1158 & 8.5378 & $2005-2014$ & (Imer et al., 2013) \\
\hline CH-Lae & MF & 47.4781 & 8.3650 & 2004-2014 & (Etzold et al., 2011) \\
\hline CH-Oe1 & GRA & 47.2858 & 7.7319 & $2002-2008$ & (Ammann et al., 2009) \\
\hline $\mathrm{CH}-\mathrm{Oe} 2$ & $\mathrm{CRO}$ & 47.2863 & 7.7343 & 2004-2014 & (Dietiker et al., 2010) \\
\hline CN-Cha & MF & 42.4025 & 128.0958 & $2003-2005$ & (Guan et al., 2006) \\
\hline CN-Cng & GRA & 44.5934 & 123.5092 & $2007-2010$ & $(? ? ?)$ \\
\hline CN-Dan & GRA & 30.4978 & 91.0664 & 2004-2005 & (Shi et al., 2006) \\
\hline CN-Din & EBF & 23.1733 & 112.5361 & $2003-2005$ & (Yan et al., 2013) \\
\hline CN-Du2 & GRA & 42.0467 & 116.2836 & $2006-2008$ & (Chen et al., 2009) \\
\hline $\mathrm{CN}-\mathrm{Ha} 2$ & WET & 37.6086 & 101.3269 & $2003-2005$ & $(? ? ?)$ \\
\hline CN-HaM & GRA & 37.3700 & 101.1800 & 2002-2004 & (Kato et al., 2006) \\
\hline CN-Qia & ENF & 26.7414 & 115.0581 & 2003-2005 & (Wen et al., 2010) \\
\hline $\mathrm{CN}-\mathrm{Sw} 2$ & GRA & 41.7902 & 111.8971 & 2010-2012 & (Shao et al., 2017) \\
\hline CZ-BK1 & ENF & 49.5021 & 18.5369 & 2004-2008 & (Acosta et al., 2013) \\
\hline CZ-BK2 & GRA & 49.4944 & 18.5429 & 2004-2006 & $(? ? ?)$ \\
\hline CZ-wet & WET & 49.0247 & 14.7704 & 2006-2014 & (Dušek et al., 2012) \\
\hline DE-Akm & WET & 53.8662 & 13.6834 & 2009-2014 & $(? ? ?)$ \\
\hline DE-Geb & $\mathrm{CRO}$ & 51.1001 & 10.9143 & 2001-2014 & (Anthoni et al., 2004) \\
\hline DE-Gri & GRA & 50.9500 & 13.5126 & $2004-2014$ & (Prescher et al., 2010a) \\
\hline DE-Hai & DBF & 51.0792 & 10.4530 & 2000-2012 & (Knohl et al., 2003) \\
\hline DE-Kli & $\mathrm{CRO}$ & 50.8931 & 13.5224 & $2004-2014$ & (Prescher et al., 2010b) \\
\hline DE-Lkb & ENF & 49.0996 & 13.3047 & 2009-2013 & (Lindauer et al., 2014) \\
\hline DE-Obe & ENF & 50.7867 & 13.7213 & $2008-2014$ & $(? ? ?)$ \\
\hline DE-RuR & GRA & 50.6219 & 6.3041 & 2011-2014 & (Post et al., 2015) \\
\hline DE-RuS & $\mathrm{CRO}$ & 50.8659 & 6.4472 & 2011-2014 & (Mauder et al., 2013) \\
\hline DE-Seh & $\mathrm{CRO}$ & 50.8706 & 6.4497 & $2007-2010$ & (Schmidt et al., 2012) \\
\hline DE-SfN & WET & 47.8064 & 11.3275 & 2012-2014 & (Hommeltenberg et al., 2014) \\
\hline DE-Spw & WET & 51.8923 & 14.0337 & 2010-2014 & $(? ? ?)$ \\
\hline DE-Tha & ENF & 50.9624 & 13.5652 & 1996-2014 & (Grünwald and Bernhofer, 2007) \\
\hline DK-Fou & $\mathrm{CRO}$ & 56.4842 & 9.5872 & $2005-2005$ & $(? ? ?)$ \\
\hline $\mathrm{DK}-\mathrm{NuF}$ & WET & 64.1308 & -51.3861 & $2008-2014$ & (Westergaard-Nielsen et al., 2013) \\
\hline DK-Sor & $\mathrm{DBF}$ & 55.4859 & 11.6446 & $1996-2014$ & (Pilegaard et al., 2011) \\
\hline $\mathrm{DK}-\mathrm{ZaF}$ & WET & 74.4814 & -20.5545 & $2008-2011$ & (Stiegler et al., 2016) \\
\hline DK-ZaH & GRA & 74.4732 & -20.5503 & $2000-2014$ & (Lund et al., 2012) \\
\hline ES-LgS & $\mathrm{OSH}$ & 37.0979 & -2.9658 & 2007-2009 & (Reverter et al., 2010) \\
\hline ES-Ln2 & $\mathrm{OSH}$ & 36.9695 & -3.4758 & 2009-2009 & (Serrano-Ortiz et al., 2011) \\
\hline FI-Hyy & ENF & 61.8474 & 24.2948 & 1996-2014 & (Suni et al., 2003) \\
\hline FI-Jok & $\mathrm{CRO}$ & 60.8986 & 23.5135 & $2000-2003$ & (Lohila, 2004) \\
\hline FI-Lom & WET & 67.9972 & 24.2092 & 2007-2009 & (Aurela et al., 2015) \\
\hline FI-Sod & ENF & 67.3619 & 26.6378 & 2001-2014 & (Thum et al., 2007) \\
\hline FR-Fon & DBF & 48.4764 & 2.7801 & $2005-2014$ & (Delpierre et al., 2015) \\
\hline FR-Gri & $\mathrm{CRO}$ & 48.8442 & 1.9519 & $2004-2013$ & (Loubet et al., 2011) \\
\hline FR-LBr & ENF & 44.7171 & -0.7693 & 1996-2008 & (Berbigier et al., 2001) \\
\hline FR-Pue & $\mathrm{EBF}$ & 43.7414 & 3.5958 & $2000-2014$ & (Rambal et al., 2004) \\
\hline GF-Guy & EBF & 5.2788 & -52.9249 & $2004-2014$ & (Bonal et al., 2008) \\
\hline IT-BCi & $\mathrm{CRO}$ & 40.5238 & 14.9574 & $2004-2014$ & (Vitale et al., 2015) \\
\hline IT-CA1 & $\mathrm{DBF}$ & 42.3804 & 12.0266 & 2011-2014 & (Sabbatini et al., 2016a) \\
\hline IT-CA2 & $\mathrm{CRO}$ & 42.3772 & 12.0260 & 2011-2014 & (Sabbatini et al., 2016b) \\
\hline IT-CA3 & DBF & 42.3800 & 12.0222 & 2011-2014 & (Sabbatini et al., 2016c) \\
\hline IT-Col & DBF & 41.8494 & 13.5881 & 1996-2014 & (Valentini et al., 1996) \\
\hline IT-Cp2 & $\mathrm{EBF}$ & 41.7043 & 12.3573 & 2012-2014 & (Fares et al., 2014) \\
\hline IT-Cpz & $\mathrm{EBF}$ & 41.7052 & 12.3761 & 1997-2009 & (Garbulsky et al., 2008) \\
\hline
\end{tabular}




\begin{tabular}{|c|c|c|c|c|c|}
\hline Site code & Veg type & Latitude & Longitude & Period & References \\
\hline IT-Isp & DBF & 45.8126 & 8.6336 & 2013-2014 & (Ferréa et al., 2012) \\
\hline IT-La2 & ENF & 45.9542 & 11.2853 & 2000-2002 & (Marcolla et al., 2003a) \\
\hline IT-Lav & ENF & 45.9562 & 11.2813 & 2003-2014 & (Marcolla et al., 2003b) \\
\hline IT-MBo & GRA & 46.0147 & 11.0458 & $2003-2013$ & (Marcolla et al., 2011) \\
\hline IT-Noe & $\mathrm{CSH}$ & 40.6061 & 8.1515 & 2004-2014 & (Papale et al., 2014) \\
\hline IT-PT1 & $\mathrm{DBF}$ & 45.2009 & 9.0610 & $2002-2004$ & (Migliavacca et al., 2009) \\
\hline IT-Ren & $\mathrm{ENF}$ & 46.5869 & 11.4337 & $1998-2013$ & (Montagnani et al., 2009) \\
\hline IT-Ro1 & $\mathrm{DBF}$ & 42.4081 & 11.9300 & $2000-2008$ & (Rey et al., 2002) \\
\hline IT-Ro2 & $\mathrm{DBF}$ & 42.3903 & 11.9209 & $2002-2012$ & (Tedeschi et al., 2006) \\
\hline IT-SR2 & $\mathrm{ENF}$ & 43.7320 & 10.2910 & $2013-2014$ & (Hoshika et al., 2017) \\
\hline IT-SRo & ENF & 43.7279 & 10.2844 & 1999-2012 & (Chiesi et al., 2005) \\
\hline IT-Tor & GRA & 45.8444 & 7.5781 & $2008-2014$ & (Galvagno et al., 2013) \\
\hline JP-MBF & $\mathrm{DBF}$ & 44.3869 & 142.3186 & $2003-2005$ & (Matsumoto et al., 2008a) \\
\hline JP-SMF & MF & 35.2617 & 137.0788 & $2002-2006$ & (Matsumoto et al., 2008b) \\
\hline NL-Hor & GRA & 52.2404 & 5.0713 & 2004-2011 & (Jacobs et al., 2007) \\
\hline NL-Loo & ENF & 52.1666 & 5.7436 & $1996-2013$ & (Moors, 2012) \\
\hline NO-Adv & WET & 78.1860 & 15.9230 & 2011-2014 & $(? ? ?)$ \\
\hline RU-Che & WET & 68.6130 & 161.3414 & $2002-2005$ & (Merbold et al., 2009a) \\
\hline RU-Cok & $\mathrm{OSH}$ & 70.8291 & 147.4943 & $2003-2014$ & (Molen et al., 2007) \\
\hline RU-Fyo & ENF & 56.4615 & 32.9221 & 1998-2014 & (Kurbatova et al., 2008) \\
\hline RU-Ha1 & GRA & 54.7252 & 90.0022 & $2002-2004$ & (Marchesini et al., 2007) \\
\hline SD-Dem & SAV & 13.2829 & 30.4783 & 2005-2009 & (Ardo et al., 2008) \\
\hline SN-Dhr & SAV & 15.4028 & -15.4322 & $2010-2013$ & (Tagesson et al., 2014) \\
\hline US-AR1 & GRA & 36.4267 & -99.4200 & 2009-2012 & (Raz-Yaseef et al., 2015b) \\
\hline US-AR2 & GRA & 36.6358 & -99.5975 & 2009-2012 & (Raz-Yaseef et al., 2015c) \\
\hline US-ARb & GRA & 35.5497 & -98.0402 & $2005-2006$ & (Raz-Yaseef et al., 2015a) \\
\hline US-ARc & GRA & 35.5465 & -98.0400 & $2005-2006$ & (Raz-Yaseef et al., 2015d) \\
\hline US-ARM & $\mathrm{CRO}$ & 36.6058 & -97.4888 & 2003-2012 & (Fischer et al., 2007) \\
\hline US-Blo & $\mathrm{ENF}$ & 38.8953 & -120.6328 & 1997-2007 & (Goldstein et al., 2000) \\
\hline US-GBT & ENF & 41.3658 & -106.2397 & 1999-2006 & (Zeller and Nikolov, 2000) \\
\hline US-GLE & ENF & 41.3665 & -106.2399 & $2004-2014$ & (Frank et al., 2014) \\
\hline US-KS2 & $\mathrm{CSH}$ & 28.6086 & -80.6715 & $2003-2006$ & (Powell et al., 2006) \\
\hline US-Los & WET & 46.0827 & -89.9792 & $2000-2014$ & (Sulman et al., 2009) \\
\hline US-Me1 & ENF & 44.5794 & -121.5000 & $2004-2005$ & (Irvine et al., 2007) \\
\hline US-Me2 & ENF & 44.4523 & -121.5574 & $2002-2014$ & (Irvine et al., 2008) \\
\hline US-Me6 & ENF & 44.3233 & -121.6078 & $2010-2014$ & (Ruehr et al., 2012) \\
\hline US-Myb & WET & 38.0498 & -121.7651 & $2010-2014$ & (Matthes et al., 2014) \\
\hline US-NR1 & ENF & 40.0329 & -105.5464 & 1998-2014 & (Monson et al., 2002) \\
\hline US-ORv & WET & 40.0201 & -83.0183 & 2011-2011 & (Morin et al., 2014) \\
\hline US-Prr & ENF & 65.1237 & -147.4876 & $2010-2013$ & (Nakai et al., 2013) \\
\hline US-SRG & GRA & 31.7894 & -110.8277 & $2008-2014$ & (Scott et al., 2015a) \\
\hline US-SRM & WSA & 31.8214 & -110.8661 & 2004-2014 & (Scott et al., 2009) \\
\hline US-Syv & $\mathrm{MF}$ & 46.2420 & -89.3477 & $2001-2014$ & (Desai et al., 2005) \\
\hline US-Ton & WSA & 38.4316 & -120.9660 & 2001-2014 & (Baldocchi et al., 2010) \\
\hline US-Tw1 & WET & 38.1074 & -121.6469 & $2012-2014$ & (Oikawa et al., 2017) \\
\hline US-Tw2 & $\mathrm{CRO}$ & 38.1047 & -121.6433 & $2012-2013$ & (Knox et al., 2016) \\
\hline US-Tw3 & $\mathrm{CRO}$ & 38.1159 & -121.6467 & $2013-2014$ & (Baldocchi et al., 2015) \\
\hline US-Tw4 & WET & 38.1030 & -121.6414 & $2013-2014$ & (Baldocchi, 2016) \\
\hline US-Twt & $\mathrm{CRO}$ & 38.1087 & -121.6530 & 2009-2014 & (Hatala et al., 2012) \\
\hline US-UMd & $\mathrm{DBF}$ & 45.5625 & -84.6975 & $2007-2014$ & (Gough et al., 2013) \\
\hline US-Var & GRA & 38.4133 & -120.9507 & $2000-2014$ & (Ma et al., 2007) \\
\hline US-WCr & $\mathrm{DBF}$ & 45.8059 & -90.0799 & $1999-2014$ & (Cook et al., 2004) \\
\hline
\end{tabular}




\begin{tabular}{llllll}
\hline Site code & Veg type & Latitude & Longitude & Period & References \\
\hline US-Whs & OSH & 31.7438 & -110.0522 & $2007-2014$ & (Scott et al., 2015b) \\
US-Wi0 & ENF & 46.6188 & -91.0814 & $2002-2002$ & (Noormets et al., 2007a) \\
US-Wi3 & DBF & 46.6347 & -91.0987 & $2002-2004$ & (Noormets et al., 2007b) \\
US-Wi4 & ENF & 46.7393 & -91.1663 & $2002-2005$ & (Noormets et al., 2007c) \\
US-Wi6 & OSH & 46.6249 & -91.2982 & $2002-2003$ & (Noormets et al., 2007d) \\
US-Wi9 & ENF & 46.6188 & -91.0814 & $2004-2005$ & (Noormets et al., 2007e) \\
US-Wkg & GRA & 31.7365 & -109.9419 & $2004-2014$ & (Scott et al., 2010) \\
ZA-Kru & SAV & -25.0197 & 31.4969 & $2000-2010$ & (Archibald et al., 2009) \\
ZM-Mon & DBF & -15.4378 & 23.2528 & $2000-2009$ & (Merbold et al., 2009b) \\
\hline
\end{tabular}

Acosta, M., Pavelka, M., Montagnani, L., Kutsch, W., Lindroth, A., Juszczak, R. and Janouš, D.: Soil surface CO2 efflux measurements in Norway spruce forests: Comparison between four different sites across Europe from boreal to alpine forest, Geoderma, 192, 295-303, doi:10.1016/j.geoderma.2012.08.027, 2013.

Ammann, C., Spirig, C., Leifeld, J. and Neftel, A.: Assessment of the nitrogen and carbon budget of two managed temperate grassland fields, Agriculture, Ecosystems \& Environment, 133(3-4), 150-162, doi:10.1016/j.agee.2009.05.006, 2009.

Anthoni, P. M., Knohl, A., Rebmann, C., Freibauer, A., Mund, M., Ziegler, W., Kolle, O. and Schulze, E.-D.: Forest and agricultural land-use-dependent CO2 exchange in Thuringia, Germany, Global Change Biology, 10(12), 2005-2019, doi:10.1111/j.1365-2486.2004.00863.x, 2004.

Archibald, S. A., Kirton, A., Merwe, M. R. van der, Scholes, R. J., Williams, C. A. and Hanan, N.: Drivers of inter-annual variability in net ecosystem exchange in a semi-arid savanna ecosystem, South Africa, Biogeosciences, 6(2), 251-266, doi:10.5194/bg-6-251-2009, 2009.

Ardo, J., Molder, M., El-Tahir, B. A. and Elkhidir, H. A. M.: Seasonal variation of carbon fluxes in a sparse savanna in semi arid Sudan, Carbon Balance and Management, 3(1), 7, doi:10.1186/1750-0680-3-7, 2008.

Aubinet, M., Chermanne, B., Vandenhaute, M., Longdoz, B., Yernaux, M. and Laitat, E.: Long term carbon dioxide exchange above a mixed forest in the Belgian Ardennes, Agricultural and Forest Meteorology, 108(4), 293-315, doi:10.1016/s0168-1923(01)00244-1, 2001.

Aurela, M., Lohila, A., Tuovinen, J. P., Hatakka, J., Penttilä, T. and Laurila, T.: Carbon dioxide and energy flux measurements in four northern-boreal ecosystems at Pallas, Boreal Environment Research, 20(4), 455-473, 2015.

Baldocchi, D.: AmeriFlux US-Tw4 Twitchell East End Wetland from 2013-present, doi:10.17190/AMF/1246151, 2016.

Baldocchi, D., Chen, Q., Chen, X., Ma, S., Miller, G., Ryu, Y., Xiao, J., Wenk, R. and Battles, J.: The dynamics of energy, water, and carbon fluxes in a blue oak (Quercus douglasii) savanna in California, in Ecosystem function in Savannas, pp. 135-151, CRC Press., 2010.

Baldocchi, D., Sturtevant, C. and Contributors, F.: Does day and night sampling reduce spurious correlation between canopy photosynthesis and ecosystem respiration?, Agricultural and Forest Meteorology, 207, 117-126, doi:10.1016/j.agrformet.2015.03.010, 2015.

Berbigier, P., Bonnefond, J.-M. and Mellmann, P.: CO2 and water vapour fluxes for 2 years above Euroflux forest site, Agricultural and Forest Meteorology, 108(3), 183-197, doi:10.1016/s0168-1923(01)00240-4, 2001.

Bergeron, O., Margolis, H. A., Black, T. A., Coursolle, C., Dunn, A. L., Barr, A. G. and Wofsy, S. C.: Comparison of carbon dioxide fluxes over three boreal black spruce forests in Canada, Global Change Biology, 13(1), 89-107, doi:10.1111/j.1365-2486.2006.01281.x, 2007.

Beringer, J., Hutley, L. B., Hacker, J. M., Neininger, B. and U, K. T. P.: Patterns and processes of carbon, water and energy cycles across northern Australian landscapes: From point to region, Agricultural and Forest 
Meteorology, 151(11), 1409-1416, doi:10.1016/j.agrformet.2011.05.003, 2011a.

Beringer, J., Hutley, L. B., Hacker, J. M., Neininger, B. and U, K. T. P.: Patterns and processes of carbon, water and energy cycles across northern Australian landscapes: From point to region, Agricultural and Forest Meteorology, 151(11), 1409-1416, doi:10.1016/j.agrformet.2011.05.003, 2011b.

Beringer, J., Hacker, J., Hutley, L. B., Leuning, R., Arndt, S. K., Amiri, R., Bannehr, L., Cernusak, L. A., Grover, S., Hensley, C., Hocking, D., Isaac, P., Jamali, H., Kanniah, K., Livesley, S., Neininger, B., U, K. T. P., Sea, W., Straten, D., Tapper, N., Weinmann, R., Wood, S. and Zegelin, S.: SPECIALSavanna patterns of energy and carbon integrated across the landscape, Bulletin of the American Meteorological Society, 92(11), 1467-1485, doi:10.1175/2011bams2948.1, 2011c.

Beringer, J., Livesley, S. J., Randle, J. and Hutley, L. B.: Carbon dioxide fluxes dominate the greenhouse gas exchanges of a seasonal wetland in the wetdry tropics of northern Australia, Agricultural and Forest Meteorology, 182-183, 239-247, doi:10.1016/j.agrformet.2013.06.008, 2013.

Beringer, J., Hutley, L. B., McHugh, I., Arndt, S. K., Campbell, D., Cleugh, H. A., Cleverly, J., Dios, V. R. de, Eamus, D., Evans, B., Ewenz, C., Grace, P., Griebel, A., Haverd, V., Hinko-Najera, N., Huete, A., Isaac, P., Kanniah, K., Leuning, R., Liddell, M. J., Macfarlane, C., Meyer, W., Moore, C., Pendall, E., Phillips, A., Phillips, R. L., Prober, S. M., Restrepo-Coupe, N., Rutledge, S., Schroder, I., Silberstein, R., Southall, P., Yee, M. S., Tapper, N. J., Gorsel, E. van, Vote, C., Walker, J. and Wardlaw, T.: An introduction to the Australian and New Zealand flux tower network OzFlux, Biogeosciences, 13(21), 5895-5916, doi:10.5194/bg-13-5895-2016, 2016a.

Beringer, J., Hutley, L. B., McHugh, I., Arndt, S. K., Campbell, D., Cleugh, H. A., Cleverly, J., Dios, V. R. de, Eamus, D., Evans, B., Ewenz, C., Grace, P., Griebel, A., Haverd, V., Hinko-Najera, N., Huete, A., Isaac, P., Kanniah, K., Leuning, R., Liddell, M. J., Macfarlane, C., Meyer, W., Moore, C., Pendall, E., Phillips, A., Phillips, R. L., Prober, S. M., Restrepo-Coupe, N., Rutledge, S., Schroder, I., Silberstein, R., Southall, P., Yee, M. S., Tapper, N. J., Gorsel, E. van, Vote, C., Walker, J. and Wardlaw, T.: An introduction to the Australian and New Zealand flux tower network OzFlux, Biogeosciences, 13(21), 5895-5916, doi:10.5194/bg-13-5895-2016, $2016 \mathrm{~b}$.

Beringer, J., Hutley, L. B., McHugh, I., Arndt, S. K., Campbell, D., Cleugh, H. A., Cleverly, J., Dios, V. R. de, Eamus, D., Evans, B., Ewenz, C., Grace, P., Griebel, A., Haverd, V., Hinko-Najera, N., Huete, A., Isaac, P., Kanniah, K., Leuning, R., Liddell, M. J., Macfarlane, C., Meyer, W., Moore, C., Pendall, E., Phillips, A., Phillips, R. L., Prober, S. M., Restrepo-Coupe, N., Rutledge, S., Schroder, I., Silberstein, R., Southall, P., Yee, M. S., Tapper, N. J., Gorsel, E. van, Vote, C., Walker, J. and Wardlaw, T.: An introduction to the Australian and New Zealand flux tower network OzFlux, Biogeosciences, 13(21), 5895-5916, doi:10.5194/bg-13-5895-2016, 2016c.

Beringer, J., Hutley, L. B., McHugh, I., Arndt, S. K., Campbell, D., Cleugh, H. A., Cleverly, J., De Dios, V. R., Eamus, D., Evans, B., Ewenz, C., Grace, P., Griebel, A., Haverd, V., Hinko-Najera, N., Huete, A., Isaac, P., Kanniah, K., Leuning, R., Liddell, M. J., MacFarlane, C., Meyer, W., Moore, C., Pendall, E., Phillips, A., Phillips, R. L., Prober, S. M., Restrepo-Coupe, N., Rutledge, S., Schroder, I., Silberstein, R., Southall, P., Sun Yee, M., Tapper, N. J., Van Gorsel, E., Vote, C., Walker, J. and Wardlaw, T.: An introduction to the Australian and New Zealand flux tower network - OzFlux, Biogeosciences, 13(21), 5895-5916, doi:10.5194/bg-13-5895-2016, 2016d.

Bonal, D., Bosc, A., Ponton, S., Goret, J.-Y., Burban, B., Gross, P., Bonnefond, J.-M., Elbers, J., Longdoz, B., Epron, D., Guehl, J.-M. and Granier, A.: Impact of severe dry season on net ecosystem exchange in the neotropical rainforest of French Guiana, Global Change Biology, 14(8), 1917-1933, doi:10.1111/j.13652486.2008.01610.x, 2008.

Bristow, M., Hutley, L. B., Beringer, J., Livesley, S. J., Edwards, A. C. and Arndt, S. K.: Quantifying the relative importance of greenhouse gas emissions from current and future savanna land use change across northern Australia, Biogeosciences Discussions, 1-47, doi:10.5194/bg-2016-191, 2016.

Carrara, A., Janssens, I. A., Yuste, J. C. and Ceulemans, R.: Seasonal changes in photosynthesis, respiration and NEE of a mixed temperate forest, Agricultural and Forest Meteorology, 126(1-2), 15-31, 
doi:10.1016/j.agrformet.2004.05.002, 2004.

Cernusak, J. B. A. L. B. H. A. N. J. T. A. L. A.: Savanna fires and their impact on net ecosystem productivity in North Australia, Global Change Biology, 13(5), 990-1004, doi:10.1111/j.1365-2486.2007.01334.x, 2007.

Cernusak, L. A., Hutley, L. B., Beringer, J., Holtum, J. A. and Turner, B. L.: Photosynthetic physiology of eucalypts along a sub-continental rainfall gradient in northern Australia, Agricultural and Forest Meteorology, 151(11), 1462-1470, doi:10.1016/j.agrformet.2011.01.006, 2011.

Chen, S., Chen, J., Lin, G., Zhang, W., Miao, H., Wei, L., Huang, J. and Han, X.: Energy balance and partition in Inner Mongolia steppe ecosystems with different land use types, Agricultural and Forest Meteorology, 149(11), 1800-1809, doi:10.1016/j.agrformet.2009.06.009, 2009.

Chiesi, M., Maselli, F., Bindi, M., Fibbi, L., Cherubini, P., Arlotta, E., Tirone, G., Matteucci, G. and Seufert, G.: Modelling carbon budget of Mediterranean forests using ground and remote sensing measurements, Agricultural and Forest Meteorology, 135(1-4), 22-34, doi:10.1016/j.agrformet.2005.09.011, 2005.

Cleverly, J., Boulain, N., Villalobos-Vega, R., Grant, N., Faux, R., Wood, C., Cook, P. G., Yu, Q., Leigh, A. and Eamus, D.: Dynamics of component carbon fluxes in a semi-arid Acacia woodland, central Australia, Journal of Geophysical Research: Biogeosciences, 118(3), 1168-1185, doi:10.1002/jgrg.20101, 2013.

Cleverly, J., Eamus, D., Van Gorsel, E., Chen, C., Rumman, R., Luo, Q., Coupe, N. R., Li, L., Kljun, N., Faux, R., Yu, Q. and Huete, A.: Productivity and evapotranspiration of two contrasting semiarid ecosystems following the 2011 global carbon land sink anomaly, Agricultural and Forest Meteorology, 220, 151-159, doi:10.1016/j.agrformet.2016.01.086, 2016.

Cook, B. D., Davis, K. J., Wang, W., Desai, A., Berger, B. W., Teclaw, R. M., Martin, J. G., Bolstad, P. V., Bakwin, P. S., Yi, C. and Heilman, W.: Carbon exchange and venting anomalies in an upland deciduous forest in northern Wisconsin, USA, Agricultural and Forest Meteorology, 126(3-4), 271-295, doi:10.1016/j.agrformet.2004.06.008, 2004.

Delpierre, N., Berveiller, D., Granda, E. and Dufrêne, E.: Wood phenology, not carbon input, controls the interannual variability of wood growth in a temperate oak forest, New Phytologist, 210(2), 459-470, doi:10.1111/nph.13771, 2015.

Desai, A. R., Bolstad, P. V., Cook, B. D., Davis, K. J. and Carey, E. V.: Comparing net ecosystem exchange of carbon dioxide between an old-growth and mature forest in the upper Midwest, USA, Agricultural and Forest Meteorology, 128(1-2), 33-55, doi:10.1016/j.agrformet.2004.09.005, 2005.

Dietiker, D., Buchmann, N. and Eugster, W.: Testing the ability of the DNDC model to predict CO2 and water vapour fluxes of a Swiss cropland site, Agriculture, Ecosystems \& Environment, 139(3), 396-401, doi:10.1016/j.agee.2010.09.002, 2010.

Donat, M. G., Lowry, A. L., Alexander, L. V., O'Gorman, P. A. and Maher, N.: More extreme precipitation in the world's dry and wet regions, Nature Climate Change, 6(5), 508-513, doi:10.1038/nclimate2941, 2016.

Dunn, A. L., Barford, C. C., Wofsy, S. C., Goulden, M. L. and Daube, B. C.: A long-term record of carbon exchange in a boreal black spruce forest: Means, responses to interannual variability, and decadal trends, Global Change Biology, 13(3), 577-590, doi:10.1111/j.1365-2486.2006.01221.x, 2007.

Dušek, J., Č́žková, H., Stellner, S., Czerný, R. and Květ, J.: Fluctuating water table affects gross ecosystem production and gross radiation use efficiency in a sedge-grass marsh, Hydrobiologia, 692(1), 57-66, doi:10.1007/s10750-012-0998-z, 2012.

Etzold, S., Ruehr, N. K., Zweifel, R., Dobbertin, M., Zingg, A., Pluess, P., Häsler, R., Eugster, W. and Buchmann, N.: The carbon balance of two contrasting mountain forest ecosystems in Switzerland: Similar annual trends, but seasonal differences, Ecosystems, 14(8), 1289-1309, doi:10.1007/s10021-011-9481-3, 2011.

Fares, S., Savi, F., Muller, J., Matteucci, G. and Paoletti, E.: Simultaneous measurements of above and below canopy ozone fluxes help partitioning ozone deposition between its various sinks in a Mediterranean Oak 
Forest, Agricultural and Forest Meteorology, 198-199, 181-191, doi:10.1016/j.agrformet.2014.08.014, 2014.

Ferréa, C., Zenone, T., Comolli, R. and Seufert, G.: Estimating heterotrophic and autotrophic soil respiration in a semi-natural forest of Lombardy, Italy, Pedobiologia, 55(6), 285-294, doi:10.1016/j.pedobi.2012.05.001, 2012.

Fischer, M. L., Billesbach, D. P., Berry, J. A., Riley, W. J. and Torn, M. S.: Spatiotemporal variations in growing season exchanges of $\mathrm{CO} 2, \mathrm{H} 2 \mathrm{O}$, and sensible heat in agricultural fields of the southern Great Plains, Earth Interactions, 11(17), 1-21, doi:10.1175/ei231.1, 2007.

Frank, J. M., Massman, W. J., Ewers, B. E., Huckaby, L. S. and Negrón, J. F.: Ecosystem CO2/H2O fluxes are explained by hydraulically limited gas exchange during tree mortality from spruce bark beetles, Journal of Geophysical Research: Biogeosciences, 119(6), 1195-1215, doi:10.1002/2013jg002597, 2014.

Galvagno, M., Wohlfahrt, G., Cremonese, E., Rossini, M., Colombo, R., Filippa, G., Julitta, T., Manca, G., Siniscalco, C., Cella, U. M. di and Migliavacca, M.: Phenology and carbon dioxide source/sink strength of a subalpine grassland in response to an exceptionally short snow season, Environmental Research Letters, 8(2), 025008, doi:10.1088/1748-9326/8/2/025008, 2013.

Garbulsky, M. F., Peñuelas, J., Papale, D. and Filella, I.: Remote estimation of carbon dioxide uptake by a Mediterranean forest, Global Change Biology, 14(12), 2860-2867, doi:10.1111/j.1365-2486.2008.01684.x, 2008.

Goldstein, A., Hultman, N., Fracheboud, J., Bauer, M., Panek, J., Xu, M., Qi, Y., Guenther, A. and Baugh, W.: Effects of climate variability on the carbon dioxide, water, and sensible heat fluxes above a ponderosa pine plantation in the Sierra Nevada (CA), Agricultural and Forest Meteorology, 101(2-3), 113-129, doi:10.1016/s0168-1923(99)00168-9, 2000.

Gough, C. M., Hardiman, B. S., Nave, L. E., Bohrer, G., Maurer, K. D., Vogel, C. S., Nadelhoffer, K. J. and Curtis, P. S.: Sustained carbon uptake and storage following moderate disturbance in a Great Lakes forest, Ecological Applications, 23(5), 1202-1215, doi:10.1890/12-1554.1, 2013.

Goulden, M. L., Winston, G. C., McMillan, A. M. S., Litvak, M. E., Read, E. L., Rocha, A. V. and Elliot, J. R.: An eddy covariance mesonet to measure the effect of forest age on land?atmosphere exchange, Global Change Biology, 12(11), 2146-2162, doi:10.1111/j.1365-2486.2006.01251.x, 2006a.

Goulden, M. L., Winston, G. C., McMillan, A. M. S., Litvak, M. E., Read, E. L., Rocha, A. V. and Elliot, J. R.: An eddy covariance mesonet to measure the effect of forest age on land?atmosphere exchange, Global Change Biology, 12(11), 2146-2162, doi:10.1111/j.1365-2486.2006.01251.x, 2006b.

Goulden, M. L., Winston, G. C., McMillan, A. M. S., Litvak, M. E., Read, E. L., Rocha, A. V. and Elliot, J. R.: An eddy covariance mesonet to measure the effect of forest age on land?atmosphere exchange, Global Change Biology, 12(11), 2146-2162, doi:10.1111/j.1365-2486.2006.01251.x, 2006c.

Goulden, M. L., Winston, G. C., McMillan, A. M. S., Litvak, M. E., Read, E. L., Rocha, A. V. and Elliot, J. R.: An eddy covariance mesonet to measure the effect of forest age on land?atmosphere exchange, Global Change Biology, 12(11), 2146-2162, doi:10.1111/j.1365-2486.2006.01251.x, 2006d.

Goulden, M. L., Winston, G. C., McMillan, A. M. S., Litvak, M. E., Read, E. L., Rocha, A. V. and Elliot, J. R.: An eddy covariance mesonet to measure the effect of forest age on land?atmosphere exchange, Global Change Biology, 12(11), 2146-2162, doi:10.1111/j.1365-2486.2006.01251.x, 2006e.

Goulden, M. L., Winston, G. C., McMillan, A. M. S., Litvak, M. E., Read, E. L., Rocha, A. V. and Elliot, J. R.: An eddy covariance mesonet to measure the effect of forest age on land?atmosphere exchange, Global Change Biology, 12(11), 2146-2162, doi:10.1111/j.1365-2486.2006.01251.x, 2006f.

Goulden, M. L., Winston, G. C., McMillan, A. M. S., Litvak, M. E., Read, E. L., Rocha, A. V. and Elliot, J. R.: An eddy covariance mesonet to measure the effect of forest age on land?atmosphere exchange, Global Change Biology, 12(11), 2146-2162, doi:10.1111/j.1365-2486.2006.01251.x, 2006g.

Grünwald, T. and Bernhofer, C.: A decade of carbon, water and energy flux measurements of an old spruce 
forest at the Anchor Station Tharandt, Tellus B, 59(3), doi:10.3402/tellusb.v59i3.17000, 2007.

Guan, D.-X., Wu, J.-B., Zhao, X.-S., Han, S.-J., Yu, G.-R., Sun, X.-M. and Jin, C.-J.: CO2 fluxes over an old, temperate mixed forest in northeastern China, Agricultural and Forest Meteorology, 137(3-4), 138-149, doi:10.1016/j.agrformet.2006.02.003, 2006.

Hatala, J. A., Detto, M., Sonnentag, O., Deverel, S. J., Verfaillie, J. and Baldocchi, D. D.: Greenhouse gas (CO2, CH4, H2O) fluxes from drained and flooded agricultural peatlands in the Sacramento-San Joaquin Delta, Agriculture, Ecosystems \& Environment, 150, 1-18, doi:10.1016/j.agee.2012.01.009, 2012.

Hinko-Najera, N., Isaac, P., Beringer, J., Gorsel, E. van, Ewenz, C., McHugh, I., Exbrayat, J.-F., Livesley, S. J. and Arndt, S. K.: Net ecosystem carbon exchange of a dry temperate eucalypt forest, Biogeosciences, 14(16), 3781-3800, doi:10.5194/bg-14-3781-2017, 2017.

Hommeltenberg, J., Schmid, H. P., Drösler, M. and Werle, P.: Can a bog drained for forestry be a stronger carbon sink than a natural bog forest?, Biogeosciences, 11(13), 3477-3493, doi:10.5194/bg-11-3477-2014, 2014.

Hoshika, Y., Fares, S., Savi, F., Gruening, C., Goded, I., De Marco, A., Sicard, P. and Paoletti, E.: Stomatal conductance models for ozone risk assessment at canopy level in two Mediterranean evergreen forests, Agricultural and Forest Meteorology, 234-235, 212-221, doi:10.1016/j.agrformet.2017.01.005, 2017.

Hutley, L. B., Beringer, J., Isaac, P. R., Hacker, J. M. and Cernusak, L. A.: A sub-continental scale living laboratory: Spatial patterns of savanna vegetation over a rainfall gradient in northern Australia, Agricultural and Forest Meteorology, 151(11), 1417-1428, doi:10.1016/j.agrformet.2011.03.002, 2011.

Imer, D., Merbold, L., Eugster, W. and Buchmann, N.: Temporal and spatial variations of soil $\mathrm{CO}_{2}, \mathrm{CH}_{4}$ and $\mathrm{N}_{2} \mathrm{O}$ fluxes at three differently managed grasslands, Biogeosciences, 10(9), 5931-5945, doi:10.5194/bg-10-5931$2013,2013$.

Irvine, J., Law, B. E. and Hibbard, K. A.: Postfire carbon pools and fluxes in semiarid ponderosa pine in central Oregon, Global Change Biology, 13(8), 1748-1760, doi:10.1111/j.1365-2486.2007.01368.x, 2007.

Irvine, J., Law, B. E., Martin, J. G. and Vickers, D.: Interannual variation in soil CO2 efflux and the response of root respiration to climate and canopy gas exchange in mature ponderosa pine, Global Change Biology, 14(12), 2848-2859, doi:10.1111/j.1365-2486.2008.01682.x, 2008.

Jacobs, C. M. J., Jacobs, A. F. G., Bosveld, F. C., Hendriks, D. M. D., Hensen, A., Kroon, P. S., Moors, E. J., Nol, L., Schrier-Uijl, A. and Veenendaal, E. M.: Variability of annual $\mathrm{CO}_{2}$ exchange from Dutch grasslands, Biogeosciences, 4(5), 803-816, doi:10.5194/bg-4-803-2007, 2007.

Kato, T., Tang, Y., Gu, S., Hirota, M., Du, M., Li, Y. and Zhao, X.: Temperature and biomass influences on interannual changes in CO2 exchange in an alpine meadow on the Qinghai-Tibetan Plateau, Global Change Biology, 12(7), 1285-1298, doi:10.1111/j.1365-2486.2006.01153.x, 2006.

Kilinc, M., Beringer, J., Hutley, L. B., Tapper, N. J. and McGuire, D. A.: Carbon and water exchange of the worlds tallest angiosperm forest, Agricultural and Forest Meteorology, 182-183, 215-224, doi:10.1016/j.agrformet.2013.07.003, 2013.

Knohl, A., Schulze, E.-D., Kolle, O. and Buchmann, N.: Large carbon uptake by an unmanaged 250year-old deciduous forest in central Germany, Agricultural and Forest Meteorology, 118(3-4), 151-167, doi:10.1016/s0168-1923(03)00115-1, 2003.

Knox, S. H., Matthes, J. H., Sturtevant, C., Oikawa, P. Y., Verfaillie, J. and Baldocchi, D.: Biophysical controls on interannual variability in ecosystem-scale CO2and CH4exchange in a California rice paddy, Journal of Geophysical Research: Biogeosciences, 121(3), 978-1001, doi:10.1002/2015jg003247, 2016.

Kurbatova, J., Li, C., Varlagin, A., Xiao, X. and Vygodskaya, N.: Modeling carbon dynamics in two adjacent spruce forests with different soil conditions in Russia, Biogeosciences, 5(4), 969-980, doi:10.5194/bg-5-969-2008, 2008.

Lindauer, M., Schmid, H., Grote, R., Mauder, M., Steinbrecher, R. and Wolpert, B.: Net ecosystem exchange over a non-cleared wind-throw-disturbed upland spruce forestMeasurements and simulations, Agricultural and 
Forest Meteorology, 197, 219-234, doi:10.1016/j.agrformet.2014.07.005, 2014.

Lohila, A.: Annual CO2 exchange of a peat field growing spring barley or perennial forage grass, Journal of Geophysical Research, 109(D18), doi:10.1029/2004jd004715, 2004.

Loubet, B., Laville, P., Lehuger, S., Larmanou, E., Fléchard, C., Mascher, N., Genermont, S., Roche, R., Ferrara, R. M., Stella, P., Personne, E., Durand, B., Decuq, C., Flura, D., Masson, S., Fanucci, O., Rampon, J.-N., Siemens, J., Kindler, R., Gabrielle, B., Schrumpf, M. and Cellier, P.: Carbon, nitrogen and greenhouse gases budgets over a four years crop rotation in northern France, Plant and Soil, 343(1-2), 109-137, doi:10.1007/s11104-011-0751-9, 2011.

Lund, M., Falk, J. M., Friborg, T., Mbufong, H. N., Sigsgaard, C., Soegaard, H. and Tamstorf, M. P.: Trends in CO2 exchange in a high Arctic tundra heath, 2000-2010, Journal of Geophysical Research: Biogeosciences, 117(G2), n/a-n/a, doi:10.1029/2011jg001901, 2012.

Ma, S., Baldocchi, D. D., Xu, L. and Hehn, T.: Inter-annual variability in carbon dioxide exchange of an oak/grass savanna and open grassland in California, Agricultural and Forest Meteorology, 147(3-4), 157-171, doi:10.1016/j.agrformet.2007.07.008, 2007.

Marchesini, L. B., Papale, D., Reichstein, M., Vuichard, N., Tchebakova, N. and Valentini, R.: Carbon balance assessment of a natural steppe of southern Siberia by multiple constraint approach, Biogeosciences Discussions, 4(1), 165-208, doi:10.5194/bgd-4-165-2007, 2007.

Marcolla, B., Pitacco, A. and Cescatti, A.: Canopy architecture and turbulence structure in a coniferous forest, Boundary-Layer Meteorology, 108(1), 39-59, doi:10.1023/a:1023027709805, 2003a.

Marcolla, B., Pitacco, A. and Cescatti, A.: Canopy architecture and turbulence structure in a coniferous forest, Boundary-Layer Meteorology, 108(1), 39-59, doi:10.1023/a:1023027709805, 2003b.

Marcolla, B., Cescatti, A., Manca, G., Zorer, R., Cavagna, M., Fiora, A., Gianelle, D., Rodeghiero, M., Sottocornola, M. and Zampedri, R.: Climatic controls and ecosystem responses drive the inter-annual variability of the net ecosystem exchange of an alpine meadow, Agricultural and Forest Meteorology, 151(9), 1233-1243, doi:10.1016/j.agrformet.2011.04.015, 2011.

Matsumoto, K., Ohta, T., Nakai, T., Kuwada, T., Daikoku, K., Iida, S., Yabuki, H., Kononov, A. V., Molen, M. K. van der, Kodama, Y., Maximov, T. C., Dolman, A. J. and Hattori, S.: Energy consumption and evapotranspiration at several boreal and temperate forests in the Far East, Agricultural and Forest Meteorology, 148(12), 1978-1989, doi:10.1016/j.agrformet.2008.09.008, 2008a.

Matsumoto, K., Ohta, T., Nakai, T., Kuwada, T., Daikoku, K., Iida, S., Yabuki, H., Kononov, A. V., Molen, M. K. van der, Kodama, Y., Maximov, T. C., Dolman, A. J. and Hattori, S.: Energy consumption and evapotranspiration at several boreal and temperate forests in the Far East, Agricultural and Forest Meteorology, 148(12), 1978-1989, doi:10.1016/j.agrformet.2008.09.008, 2008b.

Matthes, J. H., Sturtevant, C., Verfaillie, J., Knox, S. and Baldocchi, D.: Parsing the variability in CH4flux at a spatially heterogeneous wetland: Integrating multiple eddy covariance towers with high-resolution flux footprint analysis, Journal of Geophysical Research: Biogeosciences, 119(7), 1322-1339, doi:10.1002/2014jg002642, 2014.

Mauder, M., Cuntz, M., Drüe, C., Graf, A., Rebmann, C., Schmid, H. P., Schmidt, M. and Steinbrecher, R.: A strategy for quality and uncertainty assessment of long-term eddy-covariance measurements, Agricultural and Forest Meteorology, 169, 122-135, doi:10.1016/j.agrformet.2012.09.006, 2013.

McHugh, I. D., Beringer, J., Cunningham, S. C., Baker, P. J., Cavagnaro, T. R., Nally, R. M. and Thompson, R. M.: Interactions between nocturnal turbulent flux, storage and advection at an ideal eucalypt woodland site, Biogeosciences, 14(12), 3027-3050, doi:10.5194/bg-14-3027-2017, 2017.

Merbold, L., Kutsch, W. L., Corradi, C., Kolle, O., Rebmann, C., Stoy, P. C., Zimov, S. A. and Schulze, E.-D.: Artificial drainage and associated carbon fluxes $(\mathrm{CO} 2 / \mathrm{CH} 4)$ in a tundra ecosystem, Global Change Biology, 15(11), 2599-2614, doi:10.1111/j.1365-2486.2009.01962.x, 2009a.

Merbold, L., Ardö, J., Arneth, A., Scholes, R. J., Nouvellon, Y., Grandcourt, A. de, Archibald, S., Bonnefond, 
J. M., Boulain, N., Brueggemann, N., Bruemmer, C., Cappelaere, B., Ceschia, E., El-Khidir, H. A. M., El-Tahir, B. A., Falk, U., Lloyd, J., Kergoat, L., Dantec, V. L., Mougin, E., Muchinda, M., Mukelabai, M. M., Ramier, D., Roupsard, O., Timouk, F., Veenendaal, E. M. and Kutsch, W. L.: Precipitation as driver of carbon fluxes in 11 African ecosystems, Biogeosciences, 6(6), 1027-1041, doi:10.5194/bg-6-1027-2009, 2009b.

Merbold, L., Eugster, W., Stieger, J., Zahniser, M., Nelson, D. and Buchmann, N.: Greenhouse gas budget (CO2, CH4and $\mathrm{N2O}$ ) of intensively managed grassland following restoration, Global Change Biology, 20(6), 1913-1928, doi:10.1111/gcb.12518, 2014.

Meyer, W. S., Kondrlovà, E. and Koerber, G. R.: Evaporation of perennial semi-arid woodland in southeastern Australia is adapted for irregular but common dry periods, Hydrological Processes, 29(17), 3714-3726, doi:10.1002/hyp.10467, 2015.

Migliavacca, M., Meroni, M., Busetto, L., Colombo, R., Zenone, T., Matteucci, G., Manca, G. and Seufert, G.: Modeling gross primary production of agro-forestry ecosystems by assimilation of satellite-derived information in a process-based model, Sensors, 9(2), 922-942, doi:10.3390/s90200922, 2009.

Milly, P. C. D. and Dunne, K. A.: Potential evapotranspiration and continental drying, Nature Climate Change, 6(10), nclimate3046, doi:10.1038/nclimate3046, 2016.

Mkhabela, M., Amiro, B., Barr, A., Black, T., Hawthorne, I., Kidston, J., McCaughey, J., Orchansky, A., Nesic, Z., Sass, A., Shashkov, A. and Zha, T.: Comparison of carbon dynamics and water use efficiency following fire and harvesting in Canadian boreal forests, Agricultural and Forest Meteorology, 149(5), 783-794, doi:10.1016/j.agrformet.2008.10.025, 2009a.

Mkhabela, M., Amiro, B., Barr, A., Black, T., Hawthorne, I., Kidston, J., McCaughey, J., Orchansky, A., Nesic, Z., Sass, A., Shashkov, A. and Zha, T.: Comparison of carbon dynamics and water use efficiency following fire and harvesting in Canadian boreal forests, Agricultural and Forest Meteorology, 149(5), 783-794, doi:10.1016/j.agrformet.2008.10.025, 2009b.

Mkhabela, M., Amiro, B., Barr, A., Black, T., Hawthorne, I., Kidston, J., McCaughey, J., Orchansky, A., Nesic, Z., Sass, A., Shashkov, A. and Zha, T.: Comparison of carbon dynamics and water use efficiency following fire and harvesting in Canadian boreal forests, Agricultural and Forest Meteorology, 149(5), 783-794, doi:10.1016/j.agrformet.2008.10.025, 2009c.

Molen, M. K. van der, Huissteden, J. van, Parmentier, F. J. W., Petrescu, A. M. R., Dolman, A. J., Maximov, T. C., Kononov, A. V., Karsanaev, S. V. and Suzdalov, D. A.: The growing season greenhouse gas balance of a continental tundra site in the Indigirka lowlands, NE Siberia, Biogeosciences, 4(6), 985-1003, doi:10.5194/bg-4-985-2007, 2007.

Monson, R. K., Turnipseed, A. A., Sparks, J. P., Harley, P. C., Scott-Denton, L. E., Sparks, K. and Huxman, T. E.: Carbon sequestration in a high-elevation, subalpine forest, Global Change Biology, 8(5), 459-478, doi:10.1046/j.1365-2486.2002.00480.x, 2002.

Montagnani, L., Manca, G., Canepa, E., Georgieva, E., Acosta, M., Feigenwinter, C., Janous, D., Kerschbaumer, G., Lindroth, A., Minach, L., Minerbi, S., Mölder, M., Pavelka, M., Seufert, G., Zeri, M. and Ziegler, W.: A new mass conservation approach to the study of $\mathrm{CO} 2$ advection in an alpine forest, Journal of Geophysical Research, 114(D7), doi:10.1029/2008jd010650, 2009.

Moors, E.: Water Use of Forests in The Netherlands, PhD thesis, Vrije Universiteit Amsterdam., 2012.

Morin, T. H., Bohrer, G., M. Frasson, R. P. d., Naor-Azreli, L., Mesi, S., Stefanik, K. C. and Schäfer, K. V. R.: Environmental drivers of methane fluxes from an urban temperate wetland park, Journal of Geophysical Research: Biogeosciences, 119(11), 2188-2208, doi:10.1002/2014jg002750, 2014.

Moureaux, C., Debacq, A., Bodson, B., Heinesch, B. and Aubinet, M.: Annual net ecosystem carbon exchange by a sugar beet crop, Agricultural and Forest Meteorology, 139(1-2), 25-39, doi:10.1016/j.agrformet.2006.05.009, 2006.

Nakai, T., Kim, Y., Busey, R. C., Suzuki, R., Nagai, S., Kobayashi, H., Park, H., Sugiura, K. and Ito, A.: Characteristics of evapotranspiration from a permafrost black spruce forest in interior Alaska, Polar Science, 
7(2), 136-148, doi:10.1016/j.polar.2013.03.003, 2013.

Noormets, A., Chen, J. and Crow, T. R.: Age-dependent changes in ecosystem carbon fluxes in managed forests in northern Wisconsin, USA, Ecosystems, 10(2), 187-203, doi:10.1007/s10021-007-9018-y, 2007a.

Noormets, A., Chen, J. and Crow, T. R.: Age-dependent changes in ecosystem carbon fluxes in managed forests in northern Wisconsin, USA, Ecosystems, 10(2), 187-203, doi:10.1007/s10021-007-9018-y, 2007b.

Noormets, A., Chen, J. and Crow, T. R.: Age-dependent changes in ecosystem carbon fluxes in managed forests in northern Wisconsin, USA, Ecosystems, 10(2), 187-203, doi:10.1007/s10021-007-9018-y, 2007c.

Noormets, A., Chen, J. and Crow, T. R.: Age-dependent changes in ecosystem carbon fluxes in managed forests in northern Wisconsin, USA, Ecosystems, 10(2), 187-203, doi:10.1007/s10021-007-9018-y, 2007d.

Noormets, A., Chen, J. and Crow, T. R.: Age-dependent changes in ecosystem carbon fluxes in managed forests in northern Wisconsin, USA, Ecosystems, 10(2), 187-203, doi:10.1007/s10021-007-9018-y, 2007e.

Oikawa, P. Y., Jenerette, G. D., Knox, S. H., Sturtevant, C., Verfaillie, J., Dronova, I., Poindexter, C. M., Eichelmann, E. and Baldocchi, D. D.: Evaluation of a hierarchy of models reveals importance of substrate limitation for predicting carbon dioxide and methane exchange in restored wetlands, Journal of Geophysical Research: Biogeosciences, 122(1), 145-167, doi:10.1002/2016jg003438, 2017.

Papale, D., Migliavacca, M., Cremonese, E., Cescatti, A., Alberti, G., Balzarolo, M., Marchesini, L. B., Canfora, E., Casa, R., Duce, P., Facini, O., Galvagno, M., Genesio, L., Gianelle, D., Magliulo, V., Matteucci, G., Montagnani, L., Petrella, F., Pitacco, A., Seufert, G., Spano, D., Stefani, P., Vaccari, F. P. and Valentini, R.: Carbon, water and energy fluxes of terrestrial ecosystems in Italy, in The greenhouse gas balance of italy, pp. 11-45, Springer Berlin Heidelberg., 2014.

Pilegaard, K., Ibrom, A., Courtney, M. S., Hummelshøj, P. and Jensen, N. O.: Increasing net CO2 uptake by a Danish beech forest during the period from 1996 to 2009, Agricultural and Forest Meteorology, 151(7), 934-946, doi:10.1016/j.agrformet.2011.02.013, 2011.

Posse, G., Lewczuk, N., Richter, K. and Cristiano, P.: Carbon and water vapor balance in a subtropical pine plantation, iForest - Biogeosciences and Forestry, 9(5), 736-742, doi:10.3832/ifor1815-009, 2016.

Post, H., Franssen, H. J. H., Graf, A., Schmidt, M. and Vereecken, H.: Uncertainty analysis of eddy covariance $\mathrm{CO}_{2}$ flux measurements for different EC tower distances using an extended two-tower approach, Biogeosciences, 12(4), 1205-1221, doi:10.5194/bg-12-1205-2015, 2015.

Powell, T. L., Bracho, R., Li, J., Dore, S., Hinkle, C. R. and Drake, B. G.: Environmental controls over net ecosystem carbon exchange of scrub oak in central Florida, Agricultural and Forest Meteorology, 141(1), 19-34, doi:10.1016/j.agrformet.2006.09.002, 2006.

Prescher, A.-K., Grünwald, T. and Bernhofer, C.: Land use regulates carbon budgets in eastern Germany: From NEE to NBP, Agricultural and Forest Meteorology, 150(7-8), 1016-1025, doi:10.1016/j.agrformet.2010.03.008, 2010a.

Prescher, A.-K., Grünwald, T. and Bernhofer, C.: Land use regulates carbon budgets in eastern germany: From NEE to NBP, Agricultural and Forest Meteorology, 150(7-8), 1016-1025, doi:10.1016/j.agrformet.2010.03.008, $2010 \mathrm{~b}$.

Prober, S. M., Thiele, K. R., Rundel, P. W., Yates, C. J., Berry, S. L., Byrne, M., Christidis, L., Gosper, C. R., Grierson, P. F., Lemson, K., Lyons, T., Macfarlane, C., O'Connor, M. H., Scott, J. K., Standish, R. J., Stock, W. D., Etten, E. J. van, Wardell-Johnson, G. W. and Watson, A.: Facilitating adaptation of biodiversity to climate change: A conceptual framework applied to the world's largest Mediterranean-climate woodland, Climatic Change, 110(1-2), 227-248, doi:10.1007/s10584-011-0092-y, 2012.

Rambal, S., Joffre, R., Ourcival, J. M., Cavender-Bares, J. and Rocheteau, A.: The growth respiration component in eddy $\mathrm{CO} 2$ flux from a Quercus ilex mediterranean forest, Global Change Biology, 10(9), 1460-1469, doi:10.1111/j.1365-2486.2004.00819.x, 2004.

Raz-Yaseef, N., Billesbach, D. P., Fischer, M. L., Biraud, S. C., Gunter, S. A., Bradford, J. A. and Torn, M. 
S.: Vulnerability of crops and native grasses to summer drying in the U.S. Southern Great Plains, Agriculture, Ecosystems \& Environment, 213, 209-218, doi:10.1016/j.agee.2015.07.021, 2015a.

Raz-Yaseef, N., Billesbach, D. P., Fischer, M. L., Biraud, S. C., Gunter, S. A., Bradford, J. A. and Torn, M. S.: Vulnerability of crops and native grasses to summer drying in the U.S. southern Great Plains, Agriculture, Ecosystems \& Environment, 213, 209-218, doi:10.1016/j.agee.2015.07.021, 2015b.

Raz-Yaseef, N., Billesbach, D. P., Fischer, M. L., Biraud, S. C., Gunter, S. A., Bradford, J. A. and Torn, M. S.: Vulnerability of crops and native grasses to summer drying in the U.S. southern Great Plains, Agriculture, Ecosystems \& Environment, 213, 209-218, doi:10.1016/j.agee.2015.07.021, 2015c.

Raz-Yaseef, N., Billesbach, D. P., Fischer, M. L., Biraud, S. C., Gunter, S. A., Bradford, J. A. and Torn, M. S.: Vulnerability of crops and native grasses to summer drying in the U.S. southern Great Plains, Agriculture, Ecosystems \& Environment, 213, 209-218, doi:10.1016/j.agee.2015.07.021, 2015d.

Reverter, B. R., Sánchez-Cañete, E. P., Resco, V., Serrano-Ortiz, P., Oyonarte, C. and Kowalski, A. S.: Analyzing the major drivers of NEE in a Mediterranean alpine shrubland, Biogeosciences, 7(9), 2601-2611, doi:10.5194/bg-7-2601-2010, 2010.

Rey, A., Pegoraro, E., Tedeschi, V., Parri, I. D., Jarvis, P. G. and Valentini, R.: Annual variation in soil respiration and its components in a coppice oak forest in central Italy, Global Change Biology, 8(9), 851-866, doi:10.1046/j.1365-2486.2002.00521.x, 2002.

Ruehr, N. K., Martin, J. G. and Law, B. E.: Effects of water availability on carbon and water exchange in a young ponderosa pine forest: Above- and belowground responses, Agricultural and Forest Meteorology, 164, 136-148, doi:10.1016/j.agrformet.2012.05.015, 2012.

Sabbatini, S., Arriga, N., Bertolini, T., Castaldi, S., Chiti, T., Consalvo, C., Djomo, S. N., Gioli, B., Matteucci, G. and Papale, D.: Greenhouse gas balance of cropland conversion to bioenergy poplar short-rotation coppice, Biogeosciences, 13(1), 95-113, doi:10.5194/bg-13-95-2016, 2016a.

Sabbatini, S., Arriga, N., Bertolini, T., Castaldi, S., Chiti, T., Consalvo, C., Djomo, S. N., Gioli, B., Matteucci, G. and Papale, D.: Greenhouse gas balance of cropland conversion to bioenergy poplar short-rotation coppice, Biogeosciences, 13(1), 95-113, doi:10.5194/bg-13-95-2016, 2016b.

Sabbatini, S., Arriga, N., Bertolini, T., Castaldi, S., Chiti, T., Consalvo, C., Djomo, S. N., Gioli, B., Matteucci, G. and Papale, D.: Greenhouse gas balance of cropland conversion to bioenergy poplar short-rotation coppice, Biogeosciences, 13(1), 95-113, doi:10.5194/bg-13-95-2016, 2016c.

Schmidt, M., Reichenau, T., Fiener, P. and Schneider, K.: The carbon budget of a winter wheat field: An eddy covariance analysis of seasonal and inter-annual variability, Agricultural and Forest Meteorology, 165, 114-126, doi:10.1016/j.agrformet.2012.05.012, 2012.

Schroder, I., Kuske, T. and Zegelin, S.: Eddy Covariance Dataset for Arcturus (2011-2013), Geoscience Australia, Canberra., 2014.

Scott, R. L., Jenerette, G. D., Potts, D. L. and Huxman, T. E.: Effects of seasonal drought on net carbon dioxide exchange from a woody-plant-encroached semiarid grassland, Journal of Geophysical Research, 114(G4), doi:10.1029/2008jg000900, 2009.

Scott, R. L., Hamerlynck, E. P., Jenerette, G. D., Moran, M. S. and Barron-Gafford, G. A.: Carbon dioxide exchange in a semidesert grassland through drought-induced vegetation change, Journal of Geophysical Research, 115(G3), doi:10.1029/2010jg001348, 2010.

Scott, R. L., Biederman, J. A., Hamerlynck, E. P. and Barron-Gafford, G. A.: The carbon balance pivot point of southwestern U.S. semiarid ecosystems: Insights from the 21st century drought, Journal of Geophysical Research: Biogeosciences, 120(12), 2612-2624, doi:10.1002/2015jg003181, 2015a.

Scott, R. L., Biederman, J. A., Hamerlynck, E. P. and Barron-Gafford, G. A.: The carbon balance pivot point of southwestern U.S. semiarid ecosystems: Insights from the 21st century drought, Journal of Geophysical 
Research: Biogeosciences, 120(12), 2612-2624, doi:10.1002/2015jg003181, 2015b.

Serrano-Ortiz, P., Marañón-Jiménez, S., Reverter, B. R., Sánchez-Cañete, E. P., Castro, J., Zamora, R. and Kowalski, A. S.: Post-fire salvage logging reduces carbon sequestration in Mediterranean coniferous forest, Forest Ecology and Management, 262(12), 2287-2296, doi:10.1016/j.foreco.2011.08.023, 2011.

Shao, C., Chen, J., Li, L., Dong, G., Han, J., Abraha, M. and John, R.: Grazing effects on surface energy fluxes in a desert steppe on the Mongolian Plateau: Ecological Applications, 27(2), 485-502, doi:10.1002/eap.1459, 2017.

Shi, P., Sun, X., Xu, L., Zhang, X., He, Y., Zhang, D. and Yu, G.: Net ecosystem CO2 exchange and controlling factors in a steppeKobresia meadow on the Tibetan Plateau, Science in China Series D: Earth Sciences, 49(S2), 207-218, doi:10.1007/s11430-006-8207-4, 2006.

Stevens, R. M., Ewenz, C. M., Grigson, G. and Conner, S. M.: Water use by an irrigated almond orchard, Irrigation Science, 30(3), 189-200, doi:10.1007/s00271-011-0270-8, 2011.

Stiegler, C., Lund, M., Christensen, T. R., Mastepanov, M. and Lindroth, A.: Two years with extreme and little snowfall: Effects on energy partitioning and surface energy exchange in a high-Arctic tundra ecosystem, The Cryosphere, 10(4), 1395-1413, doi:10.5194/tc-10-1395-2016, 2016.

Sulman, B. N., Desai, A. R., Cook, B. D., Saliendra, N. and Mackay, D. S.: Contrasting carbon dioxide fluxes between a drying shrub wetland in northern Wisconsin, USA, and nearby forests, Biogeosciences, 6(6), 1115-1126, doi:10.5194/bg-6-1115-2009, 2009.

Suni, T., Rinne, J., Reissel, A., Altimir, N., Keronen, P., Rannik, Ü., Maso, M., Kulmala, M. and Vesala, T.: Long-term measurements of surface fluxes above a Scots pine forest in Hyytiälä, southern Finland, Boreal Environ. Res., 4, 287-301, 2003.

Tagesson, T., Fensholt, R., Guiro, I., Rasmussen, M. O., Huber, S., Mbow, C., Garcia, M., Horion, S., Sandholt, I., Holm-Rasmussen, B., Göttsche, F. M., Ridler, M.-E., Olén, N., Olsen, J. L., Ehammer, A., Madsen, M., Olesen, F. S. and Ardö, J.: Ecosystem properties of semiarid savanna grassland in West Africa and its relationship with environmental variability, Global Change Biology, 21(1), 250-264, doi:10.1111/gcb.12734, 2014.

Tedeschi, V., Rey, A., Manca, G., Valentini, R., Jarvis, P. G. and Borghetti, M.: Soil respiration in a Mediterranean oak forest at different developmental stages after coppicing, Global Change Biology, 12(1), 110-121, doi:10.1111/j.1365-2486.2005.01081.x, 2006.

Thum, T., Aalto, T., Laurila, T., Aurela, M., Kolari, P. and Hari, P.: Parametrization of two photosynthesis models at the canopy scale in a northern boreal Scots pine forest, Tellus B, 59(5), doi:10.3402/tellusb.v59i5.17066, 2007.

Ulke, A. G., Gattinoni, N. N. and Posse, G.: Analysis and modelling of turbulent fluxes in two different ecosystems in Argentina, International Journal of Environment and Pollution, 58(1/2), 52, doi:10.1504/ijep.2015.076583, 2015.

Valentini, R., Angelis, P., Matteucci, G., Monaco, R., Dore, S. and Mucnozza, G. E. S.: Seasonal net carbon dioxide exchange of a beech forest with the atmosphere, Global Change Biology, 2(3), 199-207, doi:10.1111/j.1365-2486.1996.tb00072.x, 1996.

Vitale, L., Tommasi, P. D., D’Urso, G. and Magliulo, V.: The response of ecosystem carbon fluxes to LAI and environmental drivers in a maize crop grown in two contrasting seasons, International Journal of Biometeorology, 60(3), 411-420, doi:10.1007/s00484-015-1038-2, 2015.

Wen, X. F., Wang, H. M., Wang, J. L., Yu, G. R. and Sun, X. M.: Ecosystem carbon exchanges of a subtropical evergreen coniferous plantation subjected to seasonal drought, 2003-2007, Biogeosciences, 7(1), 357-369, doi:10.5194/bg-7-357-2010, 2010.

Westergaard-Nielsen, A., Lund, M., Hansen, B. U. and Tamstorf, M. P.: Camera derived vegetation greenness index as proxy for gross primary production in a low Arctic wetland area, ISPRS Journal of Photogrammetry 
and Remote Sensing, 86, 89-99, doi:10.1016/j.isprsjprs.2013.09.006, 2013.

Wick, B., Veldkamp, E., Mello, W. Z. de, Keller, M. and Crill, P.: Nitrous oxide fluxes and nitrogen cycling along a pasture chronosequence in central Amazonia, Brazil, Biogeosciences, 2(2), 175-187, doi:10.5194/bg-2175-2005, 2005.

Wohlfahrt, G., Hammerle, A., Haslwanter, A., Bahn, M., Tappeiner, U. and Cernusca, A.: Seasonal and inter-annual variability of the net ecosystem CO2 exchange of a temperate mountain grassland: Effects of weather and management, Journal of Geophysical Research, 113(D8), doi:10.1029/2007jd009286, 2008.

WorldClim: WorldClim Version2, [online] Available from: http://worldclim.org/version2/ (Accessed 9 January 2018), 2016.

Yan, J., Zhang, Y., Yu, G., Zhou, G., Zhang, L., Li, K., Tan, Z. and Sha, L.: Seasonal and inter-annual variations in net ecosystem exchange of two old-growth forests in southern China, Agricultural and Forest Meteorology, 182-183, 257-265, doi:10.1016/j.agrformet.2013.03.002, 2013.

Yee, M. S., Pauwels, V. R., Daly, E., Beringer, J., Rüdiger, C., McCabe, M. F. and Walker, J. P.: A comparison of optical and microwave scintillometers with eddy covariance derived surface heat fluxes, Agricultural and Forest Meteorology, 213, 226-239, doi:10.1016/j.agrformet.2015.07.004, 2015.

Zeller, K. and Nikolov, N.: Quantifying simultaneous fluxes of ozone, carbon dioxide and water vapor above a subalpine forest ecosystem, Environmental Pollution, 107(1), 1-20, doi:10.1016/s0269-7491(99)00156-6, 2000.

Zielis, S., Etzold, S., Zweifel, R., Eugster, W., Haeni, M. and Buchmann, N.: NEP of a Swiss subalpine forest is significantly driven not only by current but also by previous years weather, Biogeosciences, 11(6), 1627-1635, doi:10.5194/bg-11-1627-2014, 2014. 